\title{
Universiteit
}

Leiden

The Netherlands

\section{Probing Majorana edge states with a flux qubit}

Hou, C-Y; Hassler, F.; Akhmerov, A.R.; Nilsson, J.

\section{Citation}

Hou, C. -Y., Hassler, F., Akhmerov, A. R., \& Nilsson, J. (2011). Probing Majorana edge states with a flux qubit. Physical Review B, 84(5), 054538. doi:10.1103/PhysRevB.84.054538

Version:

Not Applicable (or Unknown)

License: $\quad$ Leiden University Non-exclusive license

Downloaded from: https://hdl.handle.net/1887/61291

Note: To cite this publication please use the final published version (if applicable). 


\title{
Probing Majorana edge states with a flux qubit
}

\author{
Chang-Yu Hou, ${ }^{1}$ Fabian Hassler, ${ }^{1}$ Anton R. Akhmerov, ${ }^{1}$ and Johan Nilsson ${ }^{2}$ \\ ${ }^{1}$ Instituut-Lorentz, Universiteit Leiden, P.O. Box 9506, 2300 RA Leiden, The Netherlands \\ ${ }^{2}$ Department of Physics, University of Gothenburg, 41296 Gothenburg, Sweden \\ (Received 13 January 2011; revised manuscript received 24 May 2011; published 15 August 2011)
}

\begin{abstract}
A pair of counterpropagating Majorana edge modes appears in chiral $p$-wave superconductors and in other superconducting systems belonging to the same universality class. These modes can be described by an Ising conformal field theory. We show how a superconducting flux qubit attached to such a system couples to the two chiral edge modes via the disorder field of the Ising model. Due to this coupling, measuring the backaction of the edge states on the qubit allows us to probe the properties of Majorana edge modes.
\end{abstract}

DOI: 10.1103/PhysRevB.84.054538

PACS number(s): 74.20.Mn, 73.23.-b, 74.50.+r

\section{INTRODUCTION}

Chiral Majorana fermion edge states were originally predicted to exist in the $5 / 2$ fractional quantum Hall plateau. ${ }^{1}$ These edge states support not only neutral fermionic excitations but also more exotic edge vortices. A single edge vortex corresponds to a $\pi$ phase shift to all fermions situated to one side of it. ${ }^{2-4}$ Two edge vortices may either fuse into an edge fermion or annihilate each other, with the outcome depending on the preceding evolution of the system. In other words, the edge theory (together with the corresponding bulk theory) possesses non-Abelian statistics. ${ }^{5-8}$ This unusual physics and its potential applications to topological quantum computation are the reasons why the Majorana edge states have attracted much attention recently. ${ }^{9-14}$

Similar non-Abelian anyons and their corresponding edge states appear in superconducting systems as well. Initially it was discovered that $p$-wave superconductors support nonAbelian anyons in the bulk and chiral Majorana edge states. ${ }^{5,15,16}$ Later it was shown that depositing a conventional $s$-wave superconductor on the surface of a topological insulator while breaking time-reversal symmetry provides an alternative route to realize these non-Abelian states. ${ }^{17-19}$ Alternative proposals include substituting the topological insulator by a two-dimensional electron gas with spin-orbit coupling ${ }^{20-22}$ or by a half-metal. ${ }^{23,24}$ The realizations of Majorana edge states using $s$-wave superconductors have the following advantages: First, they rely on combining simple, well-studied ingredients. Second, the materials do not have to be extremely pure, unlike samples needed to support the fractional quantum Hall edge states. Finally, the superconducting implementations of Majorana fermions may feature a larger bulk excitation gap and may therefore be operated at higher temperatures.

The downside of the superconducting implementations of Majorana edge states is the lack of means to manipulate edge vortices. ${ }^{18,19}$ Different from the 5/2 fractional quantum Hall state, the edge vortices are not coupled to charge and thus cannot be controlled by applying voltages. ${ }^{25}$ Therefore, the standard proposal to probe the edge vortices in superconducting systems is to inject fermion excitations into the edge, to let them split into edge vortices, and finally to conclude about the behavior of the edge vortices from the detection of the fermion excitations after the subsequent fusion of edge vortices. ${ }^{18,19,25,26}$
In this paper, we propose a more direct way to manipulate and measure edge vortices using a flux qubit consisting of a superconducting ring interrupted by a Josephson junction. ${ }^{27,28}$ Our main idea is based on the following observations: First, an edge vortex is created when a superconducting vortex crosses the edge. Second, the motion of the superconducting vortices can be fully controlled by a flux qubit, since by applying a flux bias to the qubit one can tune the energy cost for a vortex being present in the superconducting ring. ${ }^{27}$ In this way, attaching a flux qubit to a system supporting Majorana edge states allows one to directly create, control, and measure edge vortices without relying on splitting and fusing fermionic excitations.

We note that our proposal is not necessarily advantageous for the purposes of topological quantum computing since quantum computing with Majorana fermions may even be realized without ever using edge states. ${ }^{28-30}$ Instead the aim of our investigation is to develop a better tool for probing the fractional excitations of the edge theory.

The paper is organized as follows. In Sec. II, we discuss a schematic setup of a system where a pair of chiral Majorana fermion edge modes couples to a flux qubit as a probe of the edge states and briefly list our main findings. In Sec. III, we review the connection between the onedimensional critical transverse-field Ising model and Majorana fermion modes. We identify the vortex-tunneling operators between two edge states as the disorder fields of the Ising model, and subsequently derive an effective Hamiltonian for the flux-qubit coupled to Majorana modes. In Sec. IV, we provide the necessary formalism for evaluating the expectation values for the flux-qubit state and qubit susceptibilities. In Sec. V and Sec. VI, we compute the qubit expectation values and the two-point qubit correlation functions in the presence of the edge state coupling, and we use these results to derive the qubit susceptibility. In Sec. VII, we analyze higher-order corrections to correlation functions of the qubit state. We summarize our results in Sec. VIII. Additionally, we provide a brief overview of the flux-qubit Hamiltonian in Appendix A. In Appendix B we reduce the flux-qubit Hamiltonian to that of a two-level system and derive the coupling between the flux-qubit and the Majorana modes. In Appendix $\mathrm{C}$, we give the form of the four point correlation function for the disorder field of the Ising model. Finally, in Appendix D, we provide the detailed derivation of the 


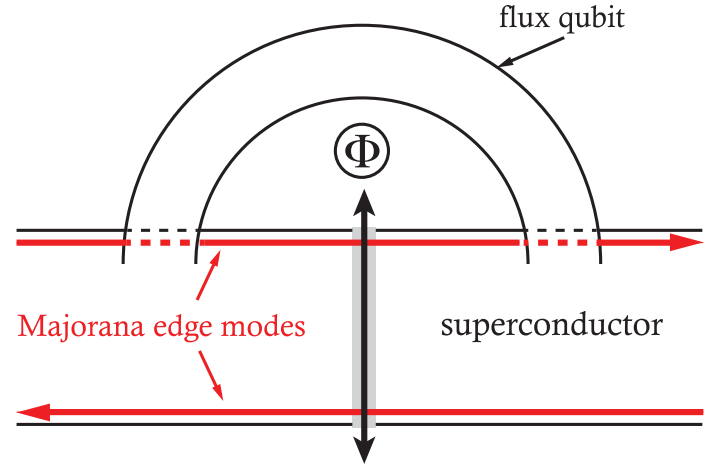

FIG. 1. (Color online) Schematic setup of the Majorana fermion edge modes coupled to a flux qubit. A pair of counterpropagating edge modes appears at two opposite edges of a topological superconductor. A flux qubit, that consists of a superconducting ring and a Josephson junction, shown as a gray rectangle, is attached to the the superconductor in such a way that it does not interrupt the edge states flow. As indicated by the arrow across the weak link, vortices can tunnel in and out of the superconducting ring through the Josephson junction.

higher order corrections to the correlation functions of the qubit state.

\section{SETUP OF THE SYSTEM}

In this paper, we consider the following setup: A strip of $s$-wave superconductor is deposited on the surface of either a three-dimensional topological insulator or a semiconductor with strong spin-orbit coupling and broken time-reversal symmetry (or any other superconducting setup supporting Majorana edge states). As depicted in Fig. 1, a pair of counterpropagating Majorana fermion edge modes appears at the two opposite edges of the superconductor. ${ }^{18,19}$ To avoid mixing between counterpropagating edge states, the width of the superconductor should be much larger than the superconducting coherence length $\hbar v_{F} / \Delta$. Here and in the following, $v_{F}$ denotes the Fermi velocity of the topological insulator (semiconductor) and $\Delta$ is the proximity-induced superconducting pairpotential. To avoid mixing of the two counterpropagating edge modes at the ends of the sample, we require the length of the superconducting strip to be longer than the dephasing length.

A flux qubit, consisting of a superconducting ring with a small inductance interrupted by a Josephson junction, is attached to the heterostructure supporting the Majorana edge modes, as shown in Fig. 1. By applying an external flux $\Phi$, the two classical states of the superconducting ring corresponding to the phase difference of 0 and $2 \pi$ across the junction can be tuned to be almost degenerate. ${ }^{27}$ In this regime, the flux qubit can be viewed as a quantum two-level system with an energy difference $\varepsilon$ (which we choose to be positive) between the states $|0\rangle$ and $|2 \pi\rangle$ and a tunneling amplitude $\delta$ between them. As described in Appendix A, the energy difference $\varepsilon$ can be easily tuned by the external flux $\Phi$ threaded through the ring.

The transition between the two qubit states is equivalent to the process of a vortex tunneling through the Josephson junction in or out of the superconducting ring. For convenience, we will refer to the Hilbert space spanned by the qubit states $|0\rangle$ and $|2 \pi\rangle$ as a spin-1/2 system. For example, we are going to call the Pauli matrices $\sigma^{x, y, z}$ acting on the qubit states the qubit spin.

A vortex tunneling through the weak link in the superconductor from one edge to the other is a phase slip of $2 \pi$ of the superconducting phase difference at the tunneling point. Due to this event, all fermions to one side of the weak link gain a phase of $\pi$. As will be shown below, the vortex-tunneling operator can be identified with the operator of the disorder field of a one-dimensional critical Ising model onto which the Majorana edge modes can be mapped.

Since vortex-tunneling events couple the qubit spin to the Majorana edge modes, we expect various observables of the qubit to carry signatures of this coupling. The main theory parameter that we are after is the scaling dimension $\Delta_{\mu}=1 / 8$ of the edge vortex operator (disorder field). Our main results apply to the regime when vortex tunneling is weak, $\varepsilon \gg \delta$.

We find that the reduction of the spin expectation value in the $z$ direction due to the vortex tunneling acquires a nontrivial scaling exponent:

$$
1-\left\langle\sigma^{z}\right\rangle \propto \frac{\delta^{2}}{\varepsilon^{2-2 \Delta_{\mu}}}=\frac{\delta^{2}}{\varepsilon^{7 / 4}} .
$$

Similarly, the spin expectation value along the $x$ direction is proportional to $\varepsilon^{2 \Delta_{\mu}-1}=\varepsilon^{-3 / 4}$, thereby probing the scaling dimension of the disorder field.

The finite-frequency susceptibilities that characterize the response of the polarization of the qubit spin to a perturbation with frequency $\omega$ provide additional information about the Majorana edge states. The susceptibility $\chi_{z z}(\omega)$, which characterizes the change of $\left\langle\sigma_{z}\right\rangle$ due to a modulation of $\sigma_{z}$ with frequency $\omega$, is measurable with current experimental techniques. It can be measured by modulating the external flux $\Phi$ and reading out the current from a dc-SQUID (SQUID is a superconducting quantum interference device) coupled to the qubit. ${ }^{31,32}$

The frequency dependence of the susceptibilities exhibits a non-Lorentzian resonant response around the frequency $\omega \approx \varepsilon$ (here and in the following, we set $\hbar=1$ ). It is modified by the coupling to the Majorana edge states and shows the scaling behavior

$$
|\chi(\omega)| \propto \frac{1}{|\omega-\varepsilon|^{1-2 \Delta_{\mu}}}=\frac{1}{|\omega-\varepsilon|^{3 / 4}},
$$

as long as $\varepsilon \gg|\omega-\varepsilon|$ and the distance $|\omega-\varepsilon|$ from the resonance is larger than the width of the resonance. The phase change of susceptibility at the resonance $\delta \phi=3 \pi / 4$ is different from the $\pi$ phase change for a usual oscillator. The origin of the extra $\pi / 4$ phase shift is the Abelian part of the statistical angle of the vortex excitations. ${ }^{9}$

\section{EDGE STATES AND COUPLING TO THE QUBIT}

\section{A. Coupling of the flux qubit to the edge states}

The flux qubit has two low-energy states, corresponding to a phase difference $\phi=0$ or $\phi=2 \pi$ across the Josephson junction at $x=x_{0}$. The Hamiltonian of the qubit is given by

$$
H_{Q}=-\frac{\varepsilon}{2} \sigma^{z}-\frac{\delta}{2} e^{i \alpha} \sigma^{+}-\frac{\delta}{2} e^{-i \alpha} \sigma^{-} .
$$


The energy difference $\varepsilon$ can be tuned by applying an external flux to the qubit while the tunneling amplitude $\delta>0$ can be manipulated by changing the Josephson coupling of the junction. ${ }^{27}$ As discussed in Appendix A, the two levels described in Eq. (3) represent the two lowest-energy states localized at the two energy minima of a double-well potential. For the two-level approximation to be accurate, the energies $\delta, \epsilon$ as well as the driving frequency $\omega$ have to be much smaller than the level spacing at each well. The tunneling phase $\alpha$ is proportional to the charge induced on the sides of the junction and its fluctuations are the main source of qubit decoherence. For simplicity we neglect the charge noise so that we can assume that $\alpha$ is static and set it to zero without loss of generality. The qubit Hamiltonian now reads

$$
H_{Q}=-\frac{\varepsilon}{2} \sigma^{z}-\frac{\delta}{2} \sigma^{x}
$$

When there is no phase difference across the Josephson junction $(\phi=0)$, the Hamiltonian of the chiral Majorana modes appearing at the edges of the superconductor, as shown in Fig. 1, reads

$$
H_{\mathrm{MF}}=\frac{i v_{M}}{2} \int \frac{d x}{2 \pi}\left[\psi_{d}(x) \partial_{x} \psi_{d}(x)-\psi_{u}(x) \partial_{x} \psi_{u}(x)\right],
$$

where $v_{M}$ is the velocity of the Majorana modes and $\psi_{u}(x)$ and $\psi_{d}(x)$ are the Majorana fermion fields at the upper and lower edges of the superconductor in Fig. 1. The sign difference between the terms containing $\psi_{u}$ and $\psi_{d}$ is due to the fact that the modes are counterpropagating. The Majorana fermion fields obey the anticommutation relations

$$
\begin{gathered}
\left\{\psi_{u}(x), \psi_{u}\left(x^{\prime}\right)\right\}=\left\{\psi_{d}(x), \psi_{d}\left(x^{\prime}\right)\right\}=2 \pi \delta\left(x-x^{\prime}\right), \\
\left\{\psi_{u}(x), \psi_{d}\left(x^{\prime}\right)\right\}=0 .
\end{gathered}
$$

A vortex tunneling through the weak link at $x=x_{0}$ advances the phase of each Cooper pair in the region $x \leqslant x_{0}$ by $2 \pi$. For Majorana fermions, just like any other fermions, this results in a phase shift of $\pi$. The effect of this phase shift is a gauge transformation

$$
H_{\mathrm{MF}} \mapsto P H_{\mathrm{MF}} P,
$$

where the parity operator $P$ is given by

$$
P=\exp \left[i \pi \int_{-\infty}^{x_{0}} d x \rho_{e}(x)\right],
$$

with the fermion density $\rho_{e}(x)=\psi^{\dagger}(x) \psi(x)$ and $\psi=\left(\psi_{u}+\right.$ $\left.i \psi_{d}\right) / 2 \sqrt{\pi}$. We refer to Appendix B for a derivation of the qubit Hamiltonian and the gauge transformation in Eq. (7). When the phase difference between two sides of the Josephson junction is exactly $\pi$, the Majorana modes approaching the junction are fully reflected. ${ }^{17}$ Since this phenomenon occurs only very close to the phase difference of $\pi$, where the system spends only a short amount of time during the process of a phase slip, we will neglect the effect of this backscattering. The relation between the phase slip and the parity operator was discussed and used in previous work focusing on the $5 / 2$ fractional quantum Hall state. , $^{3,414}$

Combining the Hamiltonian of the Majorana edge states in Eqs. (5) and (7) with the qubit Hamiltonian in Eq.(4), we get the full Hamiltonian of the coupled system in the basis of $|0\rangle$ and $|2 \pi\rangle$ :

$$
\mathcal{H}=\left(\begin{array}{cc}
H_{\mathrm{MF}} & 0 \\
0 & P H_{\mathrm{MF}} P
\end{array}\right)+H_{Q} .
$$

The first part of the Hamiltonian represents the chiral Majorana edge states coupled to the phase slip of the superconductor while the second part is the bare flux-qubit Hamiltonian.

Because the parity operator of Eq. (8) is highly nonlocal if expressed in terms of Majorana fermions, it is desirable to map the Majorana modes on a system where the vortex-tunneling event becomes a local operator. To this end, we establish the equivalence of the chiral Majorana edge modes with the longwavelength limit of the one-dimensional transverse-field Ising model at its critical point. ${ }^{33,34}$

\section{B. Mapping on the critical Ising model}

The lattice Hamiltonian of the Ising model at the critical point is given by 33,34

$$
H_{I}=-J \sum_{n}\left(s_{n}^{x} s_{n+1}^{x}+s_{n}^{z}\right),
$$

where $s_{n}^{\alpha}$ are the spin- $1 / 2$ operators at site $n$. With the JordanWigner transformation,

$$
\begin{gathered}
s_{n}^{+}=c_{n} \exp \left(i \pi \sum_{j<n} c_{j}^{\dagger} c_{j}\right), \\
s_{n}^{-}=c_{n}^{\dagger} \exp \left(i \pi \sum_{j<n} c_{j}^{\dagger} c_{j}\right), \quad s_{n}^{z}=1-2 c_{n}^{\dagger} c_{n},
\end{gathered}
$$

the Ising model of Eq. (10) can be cast in terms of fermions as

$$
H_{I}=J \sum_{n}\left[\left(c_{n}-c_{n}^{\dagger}\right)\left(c_{n+1}+c_{n+1}^{\dagger}\right)+c_{n}^{\dagger} c_{n}-c_{n} c_{n}^{\dagger}\right] .
$$

Here $s_{i}^{ \pm} \equiv\left(s_{i}^{x} \pm i s_{i}^{y}\right) / 2$ obey the usual on-site spin commutation relations while the fermions operators $c_{i}^{\dagger}$ and $c_{i}$ obey canonical anticommutation relations.

For each fermion, we introduce a pair of Majorana operators $\psi_{n}=\psi_{n}^{\dagger}$ and $\bar{\psi}_{n}=\bar{\psi}_{n}^{\dagger}$ such that

$$
c_{n}=\frac{e^{-i \pi / 4}}{2}\left(\psi_{n}+i \bar{\psi}_{n}\right) .
$$

The Majorana fermions satisfy the Clifford algebra:

$$
\left\{\psi_{m}, \psi_{n}\right\}=\left\{\bar{\psi}_{m}, \bar{\psi}_{n}\right\}=2 \delta_{m n}, \quad\left\{\psi_{m}, \bar{\psi}_{n}\right\}=0 .
$$

In terms of the Majorana operators, the Hamiltonian of Eq. (12) assumes the form

$$
\begin{aligned}
H_{I}= & -\frac{i J}{2} \sum_{n}\left(\psi_{n} \psi_{n+1}-\bar{\psi}_{n} \bar{\psi}_{n+1}\right. \\
& \left.+\psi_{n} \bar{\psi}_{n+1}-\bar{\psi}_{n} \psi_{n+1}-2 \psi_{n} \bar{\psi}_{n}\right) .
\end{aligned}
$$

In the long-wavelength limit, the Hamiltonian of Eq. (15) reduces to Eq. (5) with the identification of the continuum Majorana operators,

$$
\psi_{u}(x) \mapsto \sqrt{\frac{\pi}{a}} \psi_{n}, \quad \psi_{d}(x) \mapsto \sqrt{\frac{\pi}{a}} \bar{\psi}_{n}, \quad x \mapsto n a,
$$


and the velocity $v_{M} \mapsto 2 J a$. To complete the mapping, the bandwidth of the Ising model should be related to the cutoff energy $\Lambda$ of the linear dispersion of the Majorana edge states, $\Lambda \mapsto J$. Thereby, a pair of counterpropagating Majorana edge states, $\psi_{u}(x)$ and $\psi_{d}(x)$, can be mapped on the low energy sector of the one-dimensional transverse-field Ising model at its critical point.

For the parity operator in Eq. (8), we obtain a representation in terms of the Ising model with the following procedure: We first discretize $\int^{x_{0}} d x \rho_{e}(x)$ using the mapping of Eq. (16) and identify $x_{0} \equiv n_{0} a$ as a lattice point on the Ising model. Thereafter, we obtain an expression for the vortex tunneling operator $P$ in terms of the Ising model,

$$
P \mapsto \exp \left(i \pi \sum_{j \leqslant n_{0}} c_{j}^{\dagger} c_{j}\right)=\prod_{j \leqslant n_{0}} s_{j}^{z} \equiv \mu_{n_{0}+1 / 2}^{x},
$$

by using Eq. (13) and the Jordan-Wigner transformation of Eq. (11). Here, $\mu^{x}$ is the disorder field of the Ising model, i.e., the dual field of the spin field. ${ }^{33-36}$ The Ising Hamiltonian has a form identical to Eq. (10) when expressed through $\mu$ operators,

$$
H_{I}=-J \sum_{n}\left(\mu_{n-1 / 2}^{x} \mu_{n+1 / 2}^{x}+\mu_{n+1 / 2}^{z}\right),
$$

with $\mu_{n+1 / 2}^{z}=s_{n}^{z} s_{n+1}^{z} \cdot{ }^{37}$ We see that the parity operator is indeed a local operator in the dual description of the Ising model. After mapping on the Ising model, Eq. (7) becomes (here and in the following, we use the shortcut notation $\left.\mu=\mu^{x}\right)$

$$
P H_{\mathrm{MF}} P \mapsto \mu_{n_{0}+1 / 2} H_{I} \mu_{n_{0}+1 / 2},
$$

and the full Hamiltonian of Majorana edge states and the flux qubit of Eq. (9) maps onto

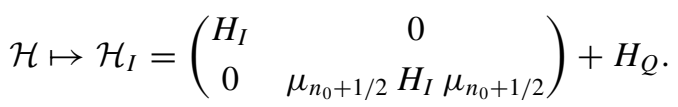

Finally, an additional unitary transformation,

$$
\begin{aligned}
\mathcal{H}_{I} & \mapsto V \mathcal{H}_{I} V^{\dagger}, \\
V=V^{\dagger} & =\left(\begin{array}{cc}
1 & 0 \\
0 & \mu_{n_{0}+1 / 2}
\end{array}\right),
\end{aligned}
$$

yields

$$
\mathcal{H}_{I}=H_{I}-\frac{\varepsilon}{2} \tau^{z}-\frac{\delta}{2} \tau^{x} \mu_{n_{0}+1 / 2}
$$

Here, $\tau^{i}$ are the Pauli matrices acting in the Hilbert space spanned by $|0\rangle$ and $\mu_{n_{0}+1 / 2}|2 \pi\rangle$. The operators of the qubit spin can be expressed through $\tau^{x, y, z}$ as

$$
\sigma^{z}=\tau^{z}, \quad \sigma^{x}=\tau^{x} \mu_{n_{0}+1 / 2}, \quad \sigma^{y}=\tau^{y} \mu_{n_{0}+1 / 2} .
$$

We use the Hamiltonian in the form of Eq. (23) and the qubit spin operators of Eqs. (24) in the rest of the paper.

The way of identifying two edge Majorana states with the complete transverse-field Ising model presented above is different from the one commonly used in preceding research. Usually, the chiral part of the Ising model is identified with a single Majorana edge. ${ }^{2,25}$ The advantages of our method are the possibility to write a complete Hamiltonian of the problem and simplified bookkeeping, while its drawback is the need for the right-moving edge and the left-moving edge to have the same geometries. Overall the differences are not important, and both methods can be used interchangeably.

\section{FORMALISM}

To probe the universal properties of Majorana edge states, the energy scales of the qubit should be much smaller than the cutoff scale of the Ising model, $\varepsilon, \delta \ll \Lambda$. In the weak coupling limit $\varepsilon \gg \delta$, we construct a perturbation theory in $\delta / \varepsilon$ by separating the Hamiltonian $\mathcal{H}_{I}=\mathcal{H}_{0}+V$ into an unperturbed part and a perturbation

$$
\mathcal{H}_{0}=H_{I}-\frac{\varepsilon}{2} \tau^{z}, \quad V=-\frac{\delta}{2} \tau^{x} \mu .
$$

Without loss of generality we set $\varepsilon>0$, so that the ground state of the unperturbed qubit is $|0\rangle$. For brevity we omit the spatial coordinate of the $\mu$ operator in the following since it is always the same in the setup that we consider.

We use the interaction picture with time-dependent operators

$$
\mathcal{O}(t)=e^{i \mathcal{H}_{0} t} \mathcal{O} e^{-i \mathcal{H}_{0} t}
$$

The perturbation $V(t)$ in this picture is given by

$$
V(t)=-\frac{\delta}{2} \mu(t)\left[\tau^{+}(t)+\tau^{-}(t)\right]
$$

where $\tau^{ \pm}(t)=e^{\mp i \varepsilon t} \tau^{ \pm}$are the time-dependent raising and lowering operators. The structure of the raising and lowering operators leads to physics similar to the Kondo and Luttinger liquid resonant tunneling problems. 2,38,39

In the calculation we need the real-time two-point and fourpoint correlation functions of $\mu$ in the long-time limit $\Lambda \mid t-$ $t^{\prime} \mid \gg 1$. The two-point correlation function is

$$
\left\langle\mu(t) \mu\left(t^{\prime}\right)\right\rangle=\frac{e^{-i \operatorname{sgn}\left(t-t^{\prime}\right) \pi / 8}}{\Lambda^{2 \Delta_{\mu}}\left|t-t^{\prime}\right|^{2 \Delta_{\mu}}},
$$

where $\operatorname{sgn}(x)$ denotes the sign of $x$ and $\Delta_{\mu}=1 / 8$ the scaling dimension of the $\mu$ field. ${ }^{40}$ The phase shift $\pi / 8$ of the two-point correlator is the Abelian part of the statistical angle for the Ising anyons braiding rules. ${ }^{9}$ Correlation functions involving a combination of multiple fields can be obtained via the underlying Ising conformal field theory or via a bosonization scheme. ${ }^{40-42}$ The expression for the four-point correlation function is given in Appendix $\mathrm{C}$ due to its length. For brevity we will measure energies in units of $\Lambda$ and times in units of $1 / \Lambda$ in the following calculation and restore the dimensionality in the final result.

We are interested in observables of the flux qubit: the spin expectation values and the spin susceptibilities. We use timedependent perturbation theory to calculate these quantities. ${ }^{43}$ This method is straightforward because of the simple form of the perturbing Hamiltonian of Eq. (27) in terms of raising and lowering operators. 
Assuming that the system is in the unperturbed ground state at time $t_{0} \rightarrow-\infty$, the expectation value of a qubit spin operator $\sigma^{\alpha}(t)$ is expressed through the $S$ matrix $S\left(t, t^{\prime}\right)$,

$$
\begin{gathered}
\left\langle\sigma^{\alpha}(t)\right\rangle=\left\langle S\left(t, t_{0}\right)^{\dagger} \sigma^{\alpha}(t) S\left(t, t_{0}\right)\right\rangle_{0}, \\
S\left(t, t^{\prime}\right)=\mathcal{T} \exp \left(-i \int_{t^{\prime}}^{t} V(s) d s\right), \quad t>t^{\prime} .
\end{gathered}
$$

Here, $\mathcal{T}$ is the time-ordering operator and $\langle\cdot\rangle_{0}$ is the expectation value with respect to the unperturbed ground state. Similarly, the two-point correlation functions of the qubit spin are given by

$$
\left\langle\sigma^{\alpha}(t) \sigma^{\beta}(0)\right\rangle=\left\langle S^{\dagger}\left(t, t_{0}\right) \sigma^{\alpha}(t) S(t, 0) \sigma^{\beta}(0) S\left(0, t_{0}\right)\right\rangle_{0} .
$$

The perturbative calculation for both the expectation values and correlation functions is done by expanding the $S$ matrices in $V$ order by order. This procedure is equivalent to the Schwinger-Keldysh formalism, with the expansion of $S$ and $S^{\dagger}$ corresponding to insertions on the forward and backward Keldysh contours.

According to linear response theory, the susceptibility is given by the Fourier transform of the retarded correlation function of the qubit: ${ }^{43}$

$$
\begin{aligned}
\chi_{\alpha \beta}(\omega) & =i \int_{0}^{\infty} d t e^{i \omega t}\left\langle\left[\sigma^{\alpha}(t), \sigma^{\beta}(0)\right]\right\rangle_{c} \\
& =-2 \int_{0}^{\infty} d t e^{i \omega t} \operatorname{Im}\left\langle\sigma^{\alpha}(t) \sigma^{\beta}(0)\right\rangle_{c}
\end{aligned}
$$

where $\langle\cdot\rangle_{c}$ denotes the cumulant,

$$
\left\langle\sigma^{\alpha}(t) \sigma^{\beta}(0)\right\rangle_{c}=\left\langle\sigma^{\alpha}(t) \sigma^{\beta}(0)\right\rangle-\left\langle\sigma^{\alpha}(t)\right\rangle\left\langle\sigma^{\beta}(0)\right\rangle,
$$

and we have used $\left\langle\sigma^{\beta}(0) \sigma^{\alpha}(t)\right\rangle_{c}=\left\langle\sigma^{\alpha}(t) \sigma^{\beta}(0)\right\rangle_{c}^{*}$. We see that to calculate the susceptibilities only the imaginary part of the correlation functions for $t>0$ is required.

\section{EXPECTATION VALUES OF THE QUBIT SPIN}

In this section, we calculate the expectation values of the qubit spin due to coupling with the Majorana edge states to the lowest nonvanishing order. Using the identity

$$
\sigma^{z}=1-2 \sigma^{-} \sigma^{+}
$$

we obtain

$$
\left\langle\sigma^{z}\right\rangle-\left\langle\sigma^{z}\right\rangle^{(0)}=-2\left\langle\sigma^{-} \sigma^{+}\right\rangle=-2\left\langle\tau^{-} \tau^{+}\right\rangle,
$$

since $\left\langle\sigma_{z}\right\rangle^{(0)}=1$.

The first nonvanishing correction in the perturbative calculation of $\left\langle\sigma^{-} \sigma^{+}\right\rangle$is of second order in $V$. By expanding $S$ and $S^{\dagger}$ in Eq. (29), we obtain

$$
\left\langle\tau^{-} \tau^{+}\right\rangle^{(2)}=\int_{-\infty}^{0} d t_{1} \int_{-\infty}^{0} d t_{2} I^{z}
$$

where

$$
I^{z}=\left\langle V\left(t_{2}\right) \tau^{-} \tau^{+} V\left(t_{1}\right)\right\rangle_{0} .
$$

The integrand $I_{z}$ originates from the first-order expansion of both $S$ and $S^{\dagger}$. The second order contributions from the same $S$ or $S^{\dagger}$ matrix vanish due to the structure of $V$ in the qubit spin space.
Substituting Eqs. (27) and (28) into the integrand $I^{z}$ yields

$$
I^{z}=\frac{\delta^{2} e^{i \varepsilon\left(t_{1}-t_{2}\right)-i \operatorname{sgn}\left(t_{2}-t_{1}\right) \pi / 8}}{4\left|t_{2}-t_{1}\right|^{2 \Delta_{\mu}}} .
$$

By evaluating the integral in Eq. (36), we find

$$
\left\langle\sigma^{z}\right\rangle^{(2)}=-2\left\langle\tau^{-} \tau^{+}\right\rangle^{(2)}=-\frac{3 \Gamma\left(\frac{3}{4}\right) \delta^{2}}{8 \varepsilon^{2-2 \Delta_{\mu}}},
$$

where $\Gamma(x)$ denotes the gamma function.

The expectation value of $\sigma^{x}$ in the unperturbed ground state vanishes. The first nonvanishing contribution to $\left\langle\sigma^{x}\right\rangle$ arises to first order in $\delta / \varepsilon$. Expanding $S$ and $S^{\dagger}$ in Eq. (29) to first order yields

$$
\left\langle\sigma^{x}\right\rangle^{(1)}=\int_{-\infty}^{0} d t_{1} I^{x}
$$

where

$$
I^{x}=-i\left\langle\left[\tau^{x} \mu(0), V\left(t_{1}\right)\right]\right\rangle_{0}=\frac{\sin \left(-\varepsilon t_{1}+\frac{\pi}{8}\right) \delta}{\left|t_{1}\right|^{2 \Delta_{\mu}}},
$$

after substituting $\sigma^{x}$ from Eqs. (24) and employing the two point correlator, Eq. (28). Evaluating Eq. (39), we find

$$
\left\langle\sigma^{x}\right\rangle^{(1)}=\frac{\Gamma\left(\frac{3}{4}\right) \delta}{\varepsilon^{1-2 \Delta_{\mu}}} .
$$

Finally, $\left\langle\sigma^{y}\right\rangle=0$ to all orders in perturbation theory since the Hamiltonian is invariant under $\sigma^{y} \mapsto-\sigma^{y}$.

\section{CORRELATION FUNCTIONS AND SUSCEPTIBILITIES OF THE FLUX-QUBIT SPIN}

Since we are interested in the behavior of susceptibilities at frequencies close to the resonance $\omega \approx \varepsilon$, we need to obtain only the long-time asymptotic of the correlation functions of the qubit spine. Using Eqs. (24) and (28), we immediately obtain that

$$
\left\langle\sigma^{x}(t) \sigma^{x}(0)\right\rangle_{c}=\frac{e^{-i \varepsilon t-i \pi / 8}}{t^{2 \Delta_{\mu}}}
$$

is nonvanishing to zeroth order. This is due to the fact that flipping the qubit spin automatically involves creation of an edge vortex, and $\sigma^{x}$ is exactly the spin-flip operator. In the same manner, one obtains that $\left\langle\sigma^{y}(t) \sigma^{y}(0)\right\rangle_{c}=\left\langle\sigma^{x}(t) \sigma^{x}(0)\right\rangle_{c}$ to zeroth order.

Concentrating next on the mixed correlator, relations (24) and (34) yield

$$
\left\langle\sigma^{x}(t) \sigma^{z}(0)\right\rangle_{c}=-2\left\langle\mu(t) \tau^{x}(t) \tau^{-}(0) \tau^{+}(0)\right\rangle_{0} .
$$

The leading nonvanishing term in this correlation function is of first order in $\delta$ and given by

$$
\left\langle\sigma^{x}(t) \sigma^{z}(0)\right\rangle_{c}^{(1)}=-\frac{\delta}{\varepsilon}\left\langle\sigma^{x}(t) \sigma^{x}(0)\right\rangle_{c}
$$

in the long-time limit.

The leading-order contribution to $\left\langle\sigma^{z}(t) \sigma^{z}(0)\right\rangle_{c}$ can be evaluated using Eq. (28) with expansions of $S$ and $S^{\dagger}$ to second order in $\delta$. In the long-time limit, the leading contribution of the correlation function is given by

$$
\left\langle\sigma^{z}(t) \sigma^{z}(0)\right\rangle_{c}^{(2)}=\frac{\delta^{2}}{\varepsilon^{2}}\left\langle\sigma^{x}(t) \sigma^{x}(0)\right\rangle_{c} .
$$


Correlators containing a single $\sigma^{y}$ vanish because of the invariance under $\sigma^{y} \mapsto-\sigma^{y}$. We see that all the nonvanishing two-point correlation functions are the same up to overall prefactors. Therefore, we will focus on $\left\langle\sigma^{x}(t) \sigma^{x}(0)\right\rangle_{c}$ in the following.

\section{A. Energy renormalization and damping}

The coupling of the flux qubit to the continuum Majorana edge states can be thought of as a two-level system coupled to an environment via interaction (27). This coupling leads to self-energy corrections $\Sigma$ for the qubit Hamiltonian,

$$
\mathcal{H}_{0} \mapsto \mathcal{H}_{0}+\Sigma, \quad \Sigma=\left(\begin{array}{cc}
\Sigma_{\uparrow \uparrow} & \Sigma_{\uparrow \downarrow} \\
\Sigma_{\downarrow \uparrow} & \Sigma_{\downarrow \downarrow}
\end{array}\right),
$$

that effectively shifts the energy spectrum and can also induce damping. ${ }^{44}$ Since we are interested only in qubit observables, we focus on the structure of $\Sigma$ for the two-level system and do not discuss the self-energy correction of the Majorana edge states.

To second order, the self-energy correction for two spin states can be written in terms of the perturbed Hamiltonian of Eqs. (25) as ${ }^{44}$

$$
\Sigma_{\alpha \beta}=\left\langle\alpha ; 0\left|V+V\left(E_{\alpha}+i 0^{+}-\mathcal{H}_{0}\right)^{-1} V\right| 0 ; \beta\right\rangle,
$$

where $E_{\alpha}$ is the energy for the spin- $\alpha=\uparrow, \downarrow$ qubit states and $|\alpha ; 0\rangle$ indicates that the Ising model is in its ground state with spin $\alpha$ for the qubit state. Due to the structure of the Hamiltonian of Eqs. (25), the first order correction to the self-energy vanishes. Additionally, the off-diagonal self-energy corrections vanish, also to second order.

By inserting a complete set $\sum_{E_{I}, \beta}\left|E_{I} ; \beta\right\rangle\left\langle\beta ; E_{I}\right|=1$ of the Hilbert space of $\mathcal{H}_{0}$, with $E_{I}$ denoting the complete set of eigenstates with energy $E_{I}$ for the Ising sector, the diagonal elements of the self-energy become

$$
\Sigma_{\alpha \alpha}=\sum_{E_{I}, \beta} \frac{\left\langle\alpha ; 0|V| E_{I} ; \beta\right\rangle\left\langle\beta ; E_{I}|V| 0 ; \alpha\right\rangle}{E_{\alpha}+i 0^{+}-\left(E_{I}+E_{\beta}\right)} .
$$

Because $V=-(\delta / 2) \tau_{x} \mu$, only terms with $\alpha \neq \beta$ give nonvanishing contributions such that

$$
\Sigma_{\alpha \alpha}=\frac{\delta^{2}}{4} \sum_{E_{I}} \frac{\left\langle 0|\mu| E_{I}\right\rangle\left\langle E_{I}|\mu| 0\right\rangle}{ \pm \varepsilon-E_{I}+i 0^{+}},
$$

where + corresponds to $\alpha=\downarrow$, and - to $\alpha=\uparrow$. The diagonal elements of the self-energy in Eq. (48) can be cast in the form

$$
\Sigma_{\alpha \alpha}=-i \frac{\delta^{2}}{4} \int_{0}^{\infty} d t e^{ \pm i \varepsilon t} e^{-0^{+} t}\langle\mu(t) \mu(0)\rangle .
$$

To see that Eq. (49) is equal to Eq. (48), we first insert a complete set of states of the Ising model, then write the time evolution of $\mu$ in the Heisenberg picture, and finally evaluate the integral.

Evaluating Eq. (49) with Eq. (28) yields

$$
\Sigma_{\uparrow \uparrow}=-\frac{\delta^{2} \Gamma\left(\frac{3}{4}\right)}{4 \varepsilon^{1-2 \Delta_{\mu}}}, \quad \Sigma_{\downarrow \downarrow}=e^{-i \pi / 4} \frac{\delta^{2} \Gamma\left(\frac{3}{4}\right)}{4 \varepsilon^{1-2 \Delta_{\mu}}},
$$

where we have used $\varepsilon>0$. The absence of the imaginary part for $\Sigma_{\uparrow \uparrow}$ indicates that the spin-up state is stable. The self-energy thus gives an energy shift to the spin-up state while it gives an energy shift with a damping to the spin-down state,

$$
E_{\alpha}= \pm \frac{\varepsilon}{2} \mapsto \pm \frac{\varepsilon}{2}+\Sigma_{\alpha \alpha}
$$

The energy renormalization and damping in Eq. (51) alter the time evolution of the ground-state correlation function

$$
\left\langle\tau^{+}(t) \tau^{-}(0)\right\rangle_{0}=e^{-i \varepsilon t} \mapsto e^{-i(\varepsilon+v) t-\gamma t / 2},
$$

where the energy renormalization and damping $v-i \gamma / 2 \equiv$ $\Sigma_{\downarrow \downarrow}-\Sigma_{\uparrow \uparrow}$ are given by

$$
v=\frac{\cos ^{2}\left(\frac{\pi}{8}\right) \Gamma\left(\frac{3}{4}\right) \delta^{2}}{2 \varepsilon^{1-2 \Delta_{\mu}}}, \quad \gamma=\frac{\Gamma\left(\frac{3}{4}\right) \delta^{2}}{2 \sqrt{2} \varepsilon^{1-2 \Delta_{\mu}}} .
$$

At zero temperature, this correlator is the only nonvanishing qubit correlator that enters in the perturbative calculation. Therefore, the effect of the self-energy can be captured by replacing

$$
\varepsilon \mapsto \varepsilon+v-\frac{i}{2} \gamma,
$$

in the qubit correlation functions computed in the longtime limit, excluding the self-energy correction. Using the replacement rule of Eq. (54), one obtains the zero- temperature correlator

$$
\left\langle\sigma^{x}(t) \sigma^{x}(0)\right\rangle_{c}=\frac{e^{-i(\varepsilon+\nu) t-\gamma t / 2-i \pi / 8}}{t^{2 \Delta_{\mu}}} .
$$

The energy renormalization and the induced damping of Eq. (51) do not arise explicitly in the lowest-order perturbation and require the resummation of the most divergent contributions to all orders in perturbation theory. In a system where Wick's theorem applies, the resummation for the self-energy can be derived explicitly from a diagrammatic perturbation scheme. ${ }^{43}$ Because the correlation functions of multiple $\mu$ 's do not obey Wick's theorem (see Appendix C), the resummation procedure for our system becomes more complicated. In the long-time limit, however, the most divergent contributions in all orders can be collected by using the operator product expansion for two $\mu$ fields that resembles the structure of Wick's theorem. ${ }^{41,42}$

\section{B. Finite temperature}

Besides $\gamma$, finite temperature is an alternative source of decoherence. The finite-temperature correlators of disorder fields are readily obtained from the zero-temperature correlators using a conformal transformation, ${ }^{45}$

$$
\frac{1}{t^{2 \Delta_{\mu}}} \mapsto \frac{\left(\pi k_{B} T\right)^{2 \Delta_{\mu}}}{\left[\sinh \left(\pi k_{B} T t\right)\right]^{2 \Delta_{\mu}}},
$$

where $T$ denotes temperature and $k_{B}$ the Boltzmann constant. The finite-temperature correlator $\left\langle\sigma^{x}(t) \sigma^{x}(0)\right\rangle_{c}$ in the longtime limit can be obtained by substituting Eq. (56) into Eq. (55) with the proviso $\varepsilon \gg k_{B} T$ such that the temperature has no direct effect on the qubit dynamics.

\section{Susceptibility}

With the correlation functions derived above, we are now in the position to evaluate susceptibilities of the qubit. We should keep in mind that these correlators are valid only in the 
long-time limit and only be used to study the behavior of only the susceptibilities close to the resonant frequency $\omega \approx \varepsilon$.

Evaluating Eq. (32) with Eq. (55) yields the susceptibility at zero temperature around the resonance,

$$
\chi_{x x}(\omega)=\frac{e^{i 3 \pi / 8} \Gamma\left(\frac{3}{4}\right)}{[i(\varepsilon+v-\omega)+\gamma / 2]^{1-2 \Delta_{\mu}}},
$$

where $v$ and $\gamma$ are given in Eqs. (53). Here, we note that the susceptibility of Eq. (57) shows non-Lorentzian response. This is in contrast to the conventional Lorentzian response of a two-level system weakly coupled to the environment. ${ }^{44,46}$ If we neglect $\nu$ and $\gamma$, which are of higher order in $\delta / \varepsilon$, this susceptibility reduces to

$$
\chi_{x x}(\omega)=\frac{\Gamma\left(\frac{3}{4}\right)}{|\omega-\varepsilon|^{1-2 \Delta_{\mu}}}\left\{\begin{array}{ll}
1, & \text { for } \omega<\varepsilon \\
e^{i 3 \pi / 4}, & \text { for } \omega>\varepsilon,
\end{array},\right.
$$

so it diverges and changes the phase by $3 \pi / 4$ at the resonant frequency. We can attribute this phase change to the phase shift of the correlator of two disorder fields in Eq. (28).

The presence of damping $\gamma$ in Eq. (57) provides a cutoff for the divergence of the response on resonance. The maximal susceptibility is reached at $\omega=\varepsilon+v$, and its value is given by

$$
\left|\chi_{x x}(\varepsilon+v)\right|=\frac{2^{1-2 \Delta_{\mu}} \Gamma\left(\frac{3}{4}\right)}{\gamma^{1-2 \Delta_{\mu}}} .
$$

Using the proportionality of correlation functions (43) and (44), one gets that $\chi_{x z}=\chi_{z x}=-(\delta / \varepsilon) \chi_{x x}$ and $\chi_{z z}=(\delta / \varepsilon)^{2} \chi_{x x}$. It is interesting to note that when $\delta \rightarrow 0$ both $\chi_{x x}$ and $\chi_{x z}$ are divergent while $\chi_{z z}$ vanishes at the resonance.

In Fig. 2, the absolute value of the susceptibility $\left|\chi_{x x}(\omega)\right|$ close to the resonance is plotted as a function of frequency. The dotted line shows the modulus of Eq. (58) for $v=\gamma=0$ while the dashed line shows that of Eq. (57). A renormalization of the resonant frequency $v$ becomes clearly visible when comparing the peak position of the dashed line with that of the dotted line.

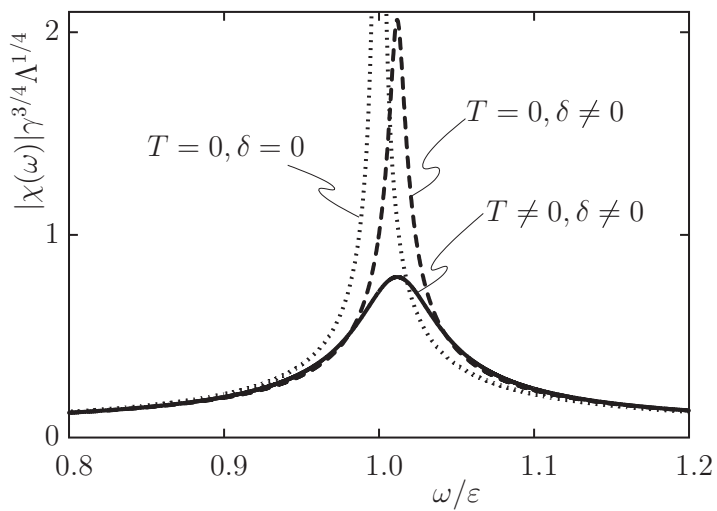

FIG. 2. Plot of the magnitude of the susceptibility $\left|\chi_{x x}(\omega)\right|$ as a function of frequency $\omega$ close to resonance $\varepsilon$. The dotted line shows the zero-temperature susceptibility in the absence of the damping and energy renormalization while the dashed line shows the result in the presence of the energy shift and the damping in Eq. (51). The parameters used for the plot are $\varepsilon=0.1 \Lambda$ and $\delta / \varepsilon=0.2$. The solid line shows a plot of the finite-temperature susceptibility with $k_{B} T=0.02 \varepsilon$.
The conformal dimension of the vortex excitation can be measured in the region with $\varepsilon \gg|\omega-\varepsilon| \gtrsim \gamma$ where

$$
\left|\chi_{x x}(\omega)\right|=\frac{\Gamma\left(\frac{3}{4}\right)}{|\omega-\varepsilon|^{1-2 \Delta_{\mu}}} .
$$

Moreover, both $\chi_{x z}$ and $\chi_{z z}$ exhibit the same scaling behavior.

The finite-temperature susceptibility of $\chi_{x x}(\omega, T)$ can be evaluated from correlation function (55) subjected to the transformation of Eq. (56). The result is plotted as the solid line in Fig. 2. An immediate effect of the temperature is that it also introduces a cutoff for the divergence on resonance. For instance, the resonance peak of the susceptibility yields a different scaling behavior with respect to the temperature,

$$
\left|\chi_{x x}(\varepsilon+\nu, T)\right| \propto T^{-\left(1-2 \Delta_{\mu}\right)},
$$

as long as $\pi k_{B} T \gg \gamma$. The zero-temperature scaling behavior of the resonance peak in Eq. (59) will be masked by a finite temperature with a crossover at $\pi k_{B} T \approx \gamma$. These scaling and crossover behaviors of the resonance strength are features of the coupling of the Majorana edge states and the flux qubit. ${ }^{46}$

The finite-temperature susceptibility shows a resonance at $\varepsilon+v$, as shown in Fig. 2. Around the resonance, the frequency dependence at finite temperature will be given by the power law in Eq. (60) but with the region constrained by $\pi k_{B} T$ instead of $\gamma$ if $\pi k_{B} T>\gamma$.

\section{HIGHER-ORDER CORRELATOR}

So far, we have computed the qubit susceptibilities to their first nonvanishing orders and the lowest-order self-energy correction $\varepsilon \mapsto \varepsilon+v-i \gamma / 2$. As a consequence, we used only the two-point correlation functions $\langle\mu(t) \mu(0)\rangle$ in our evaluations. The next nontrivial corrections to the qubit correlators involve the equal-position four-point correlator of the disorder fields $\left\langle\mu\left(t_{1}\right) \mu\left(t_{2}\right) \mu\left(t_{3}\right) \mu\left(t_{4}\right)\right\rangle$. As discussed in Appendix $C$, the four-point correlator, in principle, contains information about the non-Abelian statistics of the particles because changing the order of the fields in the correlation function not only alters the phase but can also change the functional form of the correlator. ${ }^{14}$ It is thus interesting to go beyond the lowest nonvanishing order. Additionally, doing so allows us to check the consistency of the calculation of the self-energy correction done in Sec. VIA.

As an example we focus on the second-order correction to the $\left\langle\sigma^{x}(t) \sigma^{x}(0)\right\rangle_{c}$ correlator in the long-time limit. The details of the calculation are given in Appendix D and the result in Eq. (D33). The dominant correction is a power-law divergence,

$$
\left\langle\sigma^{x}(t) \sigma^{x}(0)\right\rangle_{c}^{(2)} \propto e^{-i \pi / 8} \frac{e^{-i \varepsilon t}}{t^{2 \Delta_{\mu}}}\left[1-\left(i v+\frac{\gamma}{2}\right) t\right],
$$

which is just the second order in the $\delta$ expansion of the modified correlation function

$$
\left\langle\sigma^{x}(t) \sigma^{x}(0)\right\rangle_{c} \propto \frac{e^{-i(\varepsilon+v) t} e^{-\gamma t / 2} e^{-i \pi / 8}}{t^{2 \Delta_{\mu}}} .
$$

Hence, we confirm that the second-order perturbative correction is consistent with the the self-energy correction calculation. 
The leading correction to the susceptibility $\chi_{x x}$ in second order is due to the logarithmic term $\propto t^{-1 / 4} \log t$ in the correlator in Eq. (D33) and has the form

$$
\begin{aligned}
\chi_{x x}^{(2)}(\omega)= & -\frac{\delta^{2}(2+\sqrt{2}) \Gamma\left(\frac{7}{4}\right) \Gamma\left(\frac{3}{4}\right) e^{i 3 \pi / 8}}{\left.16 \varepsilon^{7 / 4}[i(\varepsilon+v-\omega)+\gamma / 2)\right]^{1-2 \Delta_{\mu}}} \\
& \times \ln \left(\frac{(\gamma / 2)^{2}+(\omega-\varepsilon-v)^{2}}{\varepsilon^{2}}\right)
\end{aligned}
$$

where we have included the self-energy correction of Eq. (54), and omitted terms without logarithmic divergence. Unfortunately the effects of nontrivial exchange statistics of disorder fields are not apparent in this correction.

\section{CONCLUSION AND DISCUSSION}

We have proposed a scheme to probe the edge vortex excitations of chiral Majorana fermion edge states realized in superconducting systems utilizing a flux qubit. To analyze the coupling we mapped the Hamiltonian of the Majorana edge states on the transverse-field Ising model, so that the coupling between the qubit and the Majorana edge modes becomes a local operator. In the weak coupling regime $\delta \ll \varepsilon$ we have found that the ground-state expectation values of the qubit spin are given by

$$
\left\langle\sigma^{x}\right\rangle=\frac{\Gamma\left(\frac{3}{4}\right) \delta}{\varepsilon^{1-2 \Delta_{\mu}} \Lambda^{2 \Delta_{\mu}}},\left\langle\sigma^{y}\right\rangle=0,\left\langle\sigma^{z}\right\rangle=1-\frac{3 \delta}{8 \varepsilon}\left\langle\sigma^{x}\right\rangle .
$$

Additionally, the susceptibility tensor of the qubit spin in the basis $x, y, z$ is given by

$$
\begin{gathered}
\chi(\omega)=\chi_{x x}(\omega)\left(\begin{array}{ccc}
1 & 0 & -\delta / \varepsilon \\
0 & 1 & 0 \\
-\delta / \varepsilon & 0 & (\delta / \varepsilon)^{2}
\end{array}\right), \\
\chi_{x x}(\omega)=\frac{e^{i 3 \pi / 8} \Gamma\left(\frac{3}{4}\right)}{[i(\varepsilon+v-\omega)+\gamma / 2]^{1-2 \Delta_{\mu}} \Lambda^{2 \Delta_{\mu}}},
\end{gathered}
$$

with the real part $v$ and the imaginary part $\gamma / 2$ of the selfenergy given by

$$
v=\frac{\cos ^{2}\left(\frac{\pi}{8}\right) \Gamma\left(\frac{3}{4}\right) \delta^{2}}{2 \varepsilon^{1-2 \Delta_{\mu}} \Lambda^{2 \Delta_{\mu}}}, \quad \gamma / 2=(\sqrt{2}-1) \nu .
$$

We see that all of these quantities acquire additional anomalous scaling $(\varepsilon / \Lambda)^{2 \Delta_{\mu}}$ due to the fact that each spin flip of the qubit spin couples to a disorder field $\mu$. Similar scaling with temperature appears in interferometric setups, ${ }^{25}$ but using a flux qubit allows us to attribute its origin to the dynamics of vortices much more easily and also gives additional tunability of the strength of the coupling. Another effect of the vortex tunneling being present is the phase change $\delta \phi=3 \pi / 4$ of the susceptibility around the resonance. ${ }^{46}$ This phase shift occurs due to the anomalous scaling and the presence of the Abelian statistical angle of the disorder field, in view of the fact that $\chi_{x x}$ is just a correlator of two disorder fields in the frequency domain.

The long-wavelength theory which we used is applicable only when all of the energy scales are much smaller than the cutoff energy of the Majorana modes. This is an important constraint for the flux qubit coupled to the Majorana edge states. In systems where the time-reversal symmetry is broken in the bulk (unlike for topological insulator-based proposals ${ }^{47}$ ), the velocity of the Majorana edge states can be estimated to be $v_{M} \propto v_{F} \Delta / E_{F}$ and the dispersion stays approximately linear all the way up to $\Delta$. The cutoff of the Majorana modes is related to the energy scale of the Ising model $\Lambda=\Delta \mapsto J$. Equating $J=\Delta$ and $v_{M}=2 J a$, we obtain the lattice constant of the Ising model $a=v_{F} / E_{F} \equiv \lambda_{F}$, with $\lambda_{F}$ the Fermi wavelength. The Fermi wavelength is typically smaller than any other length scale, and so the long wavelength approximation we have used is well justified. For a typical flux qubit the tunneling strength $\delta$ is indeed much smaller than the superconducting gap; the level splitting $\varepsilon$ may vary from zero to quantities much larger than the superconducting gap.

Our proposal provides a way to measure properties of the non-Abelian edge vortex excitations different from the conventional detection scheme that requires fusing vortices into fermion excitations. However, none of our results for the single flux qubit can be directly connected to the non-Abelian statistics of the quasiparticles, even after including higherorder corrections. Thus, it is of interest for future research to investigate a system where the edge vortex excitations are coupled to two qubits such that braiding of vortex excitations can be probed. ${ }^{9}$ Another feature of systems with several qubits worth investigating is the ability of the Majorana edge modes to mediate entanglement between different flux qubits.

\section{ACKNOWLEDGMENTS}

We thank C. W. J. Beenakker for useful discussions. This research was supported by the Dutch Science Foundation NWO/FOM (C.-Y. H., A. A., and F. H.) and the Swedish Research Council (Vetenskapsrådet) (J. N.).

\section{APPENDIX A: FLUX QUBIT}

The flux qubit which we consider consists of a superconducting ring interrupted by a Josephson junction which is parameterized by its critical current $I_{\mathrm{c}}$, its capacitance $C$, and the self-inductance $L$ of the ring threaded by a magnetic flux $\Phi$. The Hamiltonian in the phase basis reads ${ }^{27}$

$$
H=-4 E_{C} \frac{d^{2}}{d \phi^{2}}+E_{J}(1-\cos \phi)+\frac{E_{L}}{2}\left(\phi-2 \pi \Phi / \Phi_{0}\right)^{2},
$$

where $\phi$ is the phase difference across the Josephson junction and $\Phi_{0}=h / 2 e$ is the superconducting flux quantum. We have introduced the charging energy $E_{C}=e^{2} / 2 C$, the Josephson energy $E_{J}=\Phi_{0} I_{\mathrm{c}} / 2 \pi$, and the inductive energy $E_{L}=\Phi_{0}^{2} / 4 \pi^{2} L$.

The potential energy is given by the last two terms of the Hamiltonian of Eq. (A1). Neglecting for a moment the inductive energy, the cosine potential favors states with $\phi=$ $2 \pi \mathbb{Z}$. The transition between these state involves a change of the phase difference by $2 \pi$ which corresponds to driving a vortex in or out of the superconducting loop. The inductive energy breaks the degeneracy of the states with a different number of vortices $n$ in the loop by favoring states with $n \Phi_{0} \approx \Phi$. When the flux $\Phi$ is tuned close to $\Phi_{0} / 2$, the system becomes frustrated since the states $\phi=0$ and $\phi=2 \pi$ are then nearly degenerate in energy. When the inductive energy 
is smaller than the Josephson energy but still large enough such that states with more vortices in the superconducting loop are not accessible, the potential takes the form of a double well with the minima close to 0 and $2 \pi$. These requirements are met when $E_{L} \approx E_{J} / 2 \pi^{2}$.

The charging energy $E_{C}$ describes the influence of quantum dynamics. If the level spacing $\Omega=\sqrt{8 E_{C} E_{J}}$ in each well is large enough and additionally the two wells are well separated, only the lowest-energy states $|0\rangle$ and $|2 \pi\rangle$, which are localized near the classical minima $\phi=0,2 \pi$, are relevant. Hence, the low-energy Hamiltonian of the system reduces to Eq. (4). For $E_{L} \ll E_{J}$, the energy detuning of the two minima is given by $\varepsilon=4 \pi^{2} E_{L}\left(\frac{1}{2}-\Phi / \Phi_{0}\right)$, which can be tuned via the flux $\Phi$ in the superconducting loop. The tunneling amplitude is given by $\delta \propto \exp \left(-\sqrt{8 E_{J} / E_{C}}\right) .^{27}$

Let us now discuss the experimental parameters for the flux qubit. Assuming that the superconductor order parameter $\Delta$ is about $1 \mathrm{~K}$, the corresponding coherence length is of the order of $\xi \lesssim 1 \mu \mathrm{m}$. To avoid the mixing of the Majorana edge states, the width of Josephson junction needs to be larger than the coherence length which is in the range of micrometers. This is consistent with most experiments. ${ }^{31,48}$ Although the design of the flux qubit in Ref. 48 is more complicated than the simplest design discussed here, the idea of a $2 \pi$ phase shift for a full vortex tunneling through the Josephson junction is the same. Thus, as a concrete example, we quote the experimentally achieved parameters from Ref. 48: $E_{J} \approx 9$ $\mathrm{GHz}, E_{C} \approx 2.5 \mathrm{GHz}$, and $E_{L} \approx 0.52 \mathrm{GHz}$. The tunneling amplitude is measured and estimated to be $\delta \approx 369 \mathrm{MHz}$. $^{49}$ Moreover, the level spacing is estimated to be $\Omega=13.4 \mathrm{GHz}$.

\section{APPENDIX B: EFFECTIVE TWO-LEVEL SYSTEM}

The Euclidean action of the superconductor phase corresponding to the Hamiltonian of Eq. (A1) reads

$$
S_{\phi}=\int_{-T / 2}^{T / 2} d \tau\left[\frac{1}{2} \frac{1}{8 E_{C}} \dot{\phi}^{2}+V(\phi)\right] .
$$

As discussed in Appendix A, the double-well potential $V(\phi)$ has two energy minima located at $\phi=0$ and $2 \pi$ such that $V(\phi=0)=-\varepsilon / 2-\Omega / 2$ and $V(\phi=2 \pi)=+\varepsilon / 2-\Omega / 2$. For later convenience, we have shifted the potential energy by $\frac{1}{2} \Omega$. In our discussion, we will assume that the level spacing $\Omega=\sqrt{8 E_{J} E_{C}}$ is the same at both wells and that the potential profile connecting two minima can be approximated by $V(\phi) \sim E_{J}(1-\cos \phi)-\frac{1}{2} \Omega$. The concrete form of the potential does not affect the qualitative feature of our discussion. $^{51}$

The action of the Majorana fermions can be inferred from the Hamiltonian of Eq. (5) as

$$
S_{\psi}=\int_{-T / 2}^{T / 2} \frac{d \tau d x}{2 \pi}\left[\psi_{u} \bar{\partial} \psi_{u}+\psi_{d} \partial \psi_{d}\right]
$$

where $\partial=\left(\partial_{\tau}-i \partial_{x}\right) / 2$ and $\bar{\partial}=\left(\partial_{\tau}+i \partial_{x}\right) / 2$. The action describing the coupling between the phase field and the Majorana fermions is given by

$$
S_{\psi, \phi}=i \int_{-T / 2}^{T / 2} d \tau \frac{\dot{\phi}}{2} \int_{-\infty}^{x_{0}} d x \rho_{e}(x, \tau),
$$

where $\quad \rho_{e}(x, \tau)=\psi^{\dagger}(x, \tau) \psi(x, \tau) \quad$ and $\quad \psi(x, \tau)=$ $\left(\psi_{u}+i \psi_{d}\right) / 2 \sqrt{\pi}$ is the fermion density of the Majorana fermions. The origin of this coupling is the electrostatic energy $V Q$, where, $V=\dot{\phi} / 2$ is the voltage from the Josephson relation and $Q=\int_{-\infty}^{x_{0}} d x \rho_{e}(x, \tau)$ is the charge of the superconductor island at one side of the Josephson junction. $^{32}$ Here we have chosen a gauge such that the superconductor phase at the other side of the Josephson junction is fixed. Observe that the equation of motion of the phase field is not affected by the coupling term in Eq. (B3) when the integration of the fermion density yields no explicit time dependence.

The total action of the system thus becomes $S=S_{\phi}+$ $S_{\psi}+S_{\psi, \phi}$. From the Euclidean (imaginary-time) version of Feynman's path integral, the transition rate reads

$$
\left\langle\phi_{f}\left|e^{-H T}\right| \phi_{i}\right\rangle=\mathcal{N} \int[d \psi] \int[d \phi] e^{-S},
$$

where $H$ is the corresponding Hamiltonian, $\left|\phi_{i, f}\right\rangle$ represent the initial and final phase eigenstates, and $\mathcal{N}$ is the normalization constant. Because the leading contribution to Eq. (B4) at large times $T \rightarrow \infty$ comes from the lowest-lying energy eigenstates, the Hamiltonian at the left-hand side can be approximated by an effective Hamiltonian that contains only a few low-energy states. ${ }^{52}$

For the double-well potential $V(\phi)$, there exist two lowenergy states that are localized at the two classical minima at $\phi=0$ and $2 \pi$. By considering the transition rates within and between two minima,

$$
R_{\phi_{f}, \phi_{i}}=\left\langle\phi_{f}\left|e^{-H T}\right| \phi_{i}\right\rangle,
$$

for $\phi_{i, f}=0,2 \pi$, we would like to show that the effective Hamiltonian is a two-level system coupled to the Majorana fermions.

To compute transition rates for $\phi_{i}=\phi_{f}$, we first observe that the phase field is mostly localized at one of the wells and behaves as a simple harmonic oscillator. Therefore, the main contributions to the transition rates of Eq. (B5) come from the phase field in the localized states and are given by

$$
R_{0,0}^{0}=\sqrt{\frac{\Omega}{2}} e^{\varepsilon T / 2}, \quad R_{2 \pi, 2 \pi}^{0}=\sqrt{\frac{\Omega}{2}} e^{-\varepsilon T / 2}
$$

for the $\phi_{i}=\phi_{f}=0$ and $2 \pi$ states, respectively. ${ }^{52}$ Notice that the phase field and Majorana fermions are effectively decoupled when the phase field is localized.

The other contributions to the transition rates come from trajectories of the phase field that contain tunneling events between two wells. These tunneling events are so-called instantons and anti-instantons that occur in a very short-time interval $\Delta \tau \sim 1 / \Omega$. In the dilute- gas approximation, each instanton or anti-instanton event centered at time $\tau_{i}$ contributes to the transition rates with a factor

$$
K_{ \pm}\left(\tau_{i}\right)=\frac{\delta}{2} P_{ \pm}\left(\tau_{i}\right), \quad P_{ \pm}(\tau)=e^{ \pm i \pi \int_{-\infty}^{x_{0}} d x \rho_{e}(x, \tau)} .
$$

Here, $\delta \sim e^{-\sqrt{8 E_{J} / E_{C}}}$ is the action from the tunneling of the phase field through the barrier, and $P_{ \pm}(\tau)$ is due to the coupling of Eq. (B3) with the approximation that the time interval of instanton $\Delta \tau$ is small such that the density field can be replaced by $\rho_{e}\left(x, \tau_{i}\right)$. Since the integration of fermion density is an 
integer, we define $P_{+}(\tau)=P_{+}^{\dagger}(\tau)=P_{-}(\tau) \equiv P(\tau)$. We also note that $P^{2}(\tau)=1$. Moreover, we are not free to distribute the instantons and anti-instantons. They have to be alternated in time and the first tunneling event is determined by the initial state. $^{52}$

Let us consider the transition rate for $R_{0,0}$. Because the phase field needs to tunnel an even number of times in order to be back to the initial well, the number of (anti-)instantons has to be even for a nonvanishing contribution. A trajectory of the phase field that contains $2 n$ (anti-)instantons ordered in time, $T / 2>\tau_{2 n}>\tau_{2 n-1}>\cdots>t_{1}>-T / 2$, gives the contribution to $R_{0,0}$ as

$$
R_{0,0}^{2 n}=\sqrt{\frac{\Omega}{2}} e^{\varepsilon T}\left\langle\int \prod_{i=1}^{2 n} d \tau_{i} \frac{\delta}{2} e^{(-1)^{i+1} \varepsilon \tau_{i}} P\left(\tau_{i}\right)\right\rangle_{\psi},
$$

which is integrated over the centers of (anti-)instantons $\tau_{i}$. Here $\langle\cdots\rangle_{\psi}$ is the path integral summation over fermion fields such that

$$
\left\langle O\left(\psi_{u}, \psi_{d}\right)\right\rangle_{\psi}=\int[d \psi] O\left(\psi_{u}, \psi_{d}\right) e^{-S_{\psi}}
$$

for an arbitrary fermion field combination $O\left(\psi_{u}, \psi_{d}\right)$. The total transition rate can be written as

$$
R_{0,0}=\sum_{j=0}^{\infty} R_{0,0}^{2 j}
$$

where $R_{0,0}^{0}$ is defined in Eqs. (B6). The transition rate of $R_{2 \pi, 2 \pi}$ can be derived in the same manner and takes the same form as $R_{0,0}$ in Eq. (B10) by summing over $R_{2 \pi, 2 \pi}^{2 j}=R_{0,0}^{2 j}[\varepsilon \rightarrow-\varepsilon]$.

The transition rates between two wells, $R_{2 \pi, 0}$ and $R_{0,2 \pi}$, can also be computed by properly counting the (anti-)instanton events. The crucial difference is now that an odd number of tunneling events are needed for the final state to be in a different well than the initial state. The total transition rate of $R_{2 \pi, 0}$ then reads

$$
R_{2 \pi, 0}=\sum_{j=0}^{\infty} R_{2 \pi, 0}^{2 j+1},
$$

by summing over contributions from trajectories with odd tunneling events

$$
R_{2 \pi, 0}^{2 n+1}=\sqrt{\frac{\Omega}{2}}\left\langle\int \prod_{i=1}^{2 n+1} d \tau_{i} \frac{\delta}{2} e^{(-1)^{i+1} \varepsilon \tau_{i}} P\left(\tau_{i}\right)\right\rangle_{\psi} .
$$

Finally, $R_{0,2 \pi}$ also takes the same form as $R_{2 \pi, 0}$ with a substitution of $R_{0,2 \pi}^{2 j+1}=R_{2 \pi, 0}^{2 j+1}[\varepsilon \rightarrow-\varepsilon]$ in Eq. (B12).

By using the interaction picture, we can explicitly show that the effective Hamiltonian

$$
H_{\text {eff }}=H_{\mathrm{MF}}-\frac{\varepsilon}{2} \tau^{z}-\frac{\delta}{2} \tau^{x} P,
$$

reproduces the transition rates within and between two wells in Eqs. (B10), up to an overall constant; see Eq. (23). Here, $H_{\mathrm{MF}}$ is defined in Eq. (5) and $\tau^{x, z}$ are Pauli matrix acting on the two-level basis $|\phi\rangle, \phi \in\{0,2 \pi\}$, of the superconducting phase difference $\phi .{ }^{53}$ Note that $\tau_{x}$ enters in the Hamiltonian of Eq. (B13) together with $P$. This is a consequence of gauge invariance - whenever the superconducting phase difference changes by $2 \pi$ the phase of the Majorana to the left of the junction has to be changed by $\pi$. Every physical observable has to be gauge invariant, which is why $\tau_{x}$ (and $\tau_{y}$ for that matter) always has to occur together with $P$. In this spirit, we define the (observable) qubit degrees of freedom as

$$
\sigma^{z}=\tau^{z}, \quad \sigma^{x}=\tau^{x} P, \quad \sigma^{y}=\tau^{y} P,
$$

see Eqs. (24). We thus conclude that the two-level Hamiltonian of Eq. (B13) together with the identification of Eqs. (B14) give the effective low-energy description of the system.

\section{APPENDIX C: CORRELATION FUNCTIONS OF DISORDER FIELDS}

The one-dimensional critical transverse-field Ising model is a conformal field theory (CFT) with central charge $c=1 / 2$. This CFT contains the following primary fields: $\mathbb{1}, \epsilon=i \psi \bar{\psi}$, $s$, and $\mu$. Here $\mathbb{1}$ is the identity operator, $\epsilon$ is the energy field (a product of the right- and left-moving Majorana fermion fields $\psi$ and $\bar{\psi}$ ), and $s$ is the Ising spin field with its dual field $\mu .^{40,41}$ The dual field $\mu$ is also called the disorder field and has the same scaling behavior as the Ising spin field $s$ at the critical point. On the lattice, the disorder fields $\mu$ are nonlinear combinations of Ising spin fields $s$ and reside on the bonds of the lattice Ising model. They are hence not independent of the Ising spin field $s$.

In the continuum and in imaginary time, the two-point correlation function of disorder fields $\mu$ can be obtained from $\mathrm{CFT}^{41}$,

$$
\left\langle\mu\left(z_{1}, \bar{z}_{1}\right) \mu\left(z_{2}, \bar{z}_{2}\right)\right\rangle=\frac{1}{\left[\left(z_{1}-z_{2}\right)\left(\bar{z}_{1}-\bar{z}_{2}\right)\right]^{\Delta_{\mu}}},
$$

with $z_{i}=\tau_{i}+i x_{i}$ and $\bar{z}_{i}=\tau_{i}-i x_{i}$.

Following Ref. 14, the real-time correlators can be obtained by analytical continuation $\tau \rightarrow \xi+i t$. Here $\xi \rightarrow 0^{+}$is introduced to ensure the correct phase counting and is important for the Abelian part of the statistics. The equal position two-point correlation function is given by

$$
\left\langle\mu\left(t_{1}, x_{0}\right) \mu\left(t_{2}, x_{0}\right)\right\rangle=\frac{1}{\left(\xi+i\left(t_{1}-t_{2}\right)\right)^{2 \Delta_{\mu}}} .
$$

By using the identity

$$
\lim _{\xi \rightarrow 0^{+}} \frac{1}{(\xi+i t)^{1 / 4}}=\frac{e^{-i \operatorname{sgn}(t) \pi / 8}}{|t|^{1 / 4}},
$$

one obtains the two-point correlation function in the form of Eq. (28).

The four-point correlation function of $\mu$ 's can be obtained in a similar manner. In imaginary time, the correlation function is given by ${ }^{41}$

$$
\begin{aligned}
& \left\langle\mu\left(z_{1}, \bar{z}_{1}\right) \mu\left(z_{2}, \bar{z}_{2}\right) \mu\left(z_{3}, \bar{z}_{3}\right) \mu\left(z_{4}, \bar{z}_{4}\right)\right\rangle^{2} \\
& \quad=\left|\frac{z_{13} z_{24}}{z_{12} z_{34} z_{14} z_{23}}\right|^{1 / 2}\left(\frac{1+|\chi|+|1-\chi|}{2}\right),
\end{aligned}
$$

where $\chi=\left(z_{12} z_{34} / z_{13} z_{24}\right)$ is the conformally invariant cross ratio and the absolute values should be understood as $\left|z_{i j}\right|^{\alpha}=$ $\left(z_{i j} \bar{z}_{i j}\right)^{\alpha / 2}$. Because we are interested in tunneling at a single point, we can set $x_{i}=0$. In this limit the four-point correlation function can be evaluated to be 


$$
\left\langle\mu\left(z_{1}\right) \mu\left(z_{2}\right) \mu\left(z_{3}\right) \mu\left(z_{4}\right)\right\rangle^{2}= \begin{cases}\left|\frac{z_{13} z_{24}}{z_{12} z_{34} z_{14} z_{23}}\right|^{1 / 2}, & \text { for } 0<\chi<1 \\ \left|\frac{z_{13} z_{24}}{z_{12} z_{34} z_{14} z_{23}}\right|^{1 / 2}|1-\chi|=\left|\frac{z_{14} z_{23}}{z_{12} z_{34} z_{13} z_{24}}\right|^{1 / 2}, & \text { for } \quad \chi<0 \\ \left|\frac{z_{13} z_{24}}{z_{12} z_{34} z_{14} z_{23}}\right|^{1 / 2}|\chi|=\left|\frac{z_{12} z_{34}}{z_{14} z_{23} z_{13} z_{24}}\right|^{1 / 2}, & \text { for } \quad \chi>1\end{cases}
$$

The real-time correlation function can be obtained by first taking a square root of Eq. (C5) followed by the analytical continuation, $\tau_{i} \rightarrow \xi+i t_{i},:^{14}$

$$
\begin{aligned}
\left\langle\mu\left(t_{1}\right) \mu\left(t_{2}\right) \mu\left(t_{3}\right) \mu\left(t_{4}\right)\right\rangle= & F_{12}\left(t_{1}, t_{2}, t_{3}, t_{4}\right)[\theta(1324)+\theta(1423)+\theta(2413)+\theta(2314)+\theta(3241)+\theta(3142)+\theta(4132) \\
& +\theta(4231)]+F_{13}\left(t_{1}, t_{2}, t_{3}, t_{4}\right)[\theta(1234)+\theta(1432)+\theta(2143)+\theta(2341)+\theta(3214)+\theta(3412) \\
& +\theta(4123)+\theta(4321)]+F_{14}\left(t_{1}, t_{2}, t_{3}, t_{4}\right)[\theta(1243)+\theta(1342)+\theta(2134)+\theta(2431) \\
& +\theta(3124)+\theta(3421)+\theta(4213)+\theta(4312)]
\end{aligned}
$$

where $\theta(a b c d)=1$ for $t_{a}>t_{b}>t_{c}>t_{d}$ and is otherwise zero. The corresponding functions $F_{i j}$ are given by

$$
\begin{aligned}
& F_{12}\left(t_{1}, t_{2}, t_{3}, t_{4}\right)=\frac{\left[\xi+i\left(t_{1}-t_{2}\right)\right]^{1 / 4}\left[\xi+i\left(t_{3}-t_{4}\right)\right]^{1 / 4}}{\left[\xi+i\left(t_{1}-t_{3}\right)\right]^{1 / 4}\left[\xi+i\left(t_{1}-t_{4}\right)\right]^{1 / 4}\left[\xi+i\left(t_{2}-t_{3}\right)\right]^{1 / 4}\left[\xi+i\left(t_{2}-t_{4}\right)\right]^{1 / 4}}, \\
& F_{13}\left(t_{1}, t_{2}, t_{3}, t_{4}\right)=\frac{\left[\xi+i\left(t_{1}-t_{3}\right)\right]^{1 / 4}\left[\xi+i\left(t_{2}-t_{4}\right)\right]^{1 / 4}}{\left[\xi+i\left(t_{1}-t_{2}\right)\right]^{1 / 4}\left[\xi+i\left(t_{1}-t_{4}\right)\right]^{1 / 4}\left[\xi+i\left(t_{2}-t_{3}\right)\right]^{1 / 4}\left[\xi+i\left(t_{3}-t_{4}\right)\right]^{1 / 4}}, \\
& F_{14}\left(t_{1}, t_{2}, t_{3}, t_{4}\right)=\frac{\left[\xi+i\left(t_{1}-t_{4}\right)\right]^{1 / 4}\left[v+i\left(t_{2}-t_{3}\right)\right]^{1 / 4}}{\left[\xi+i\left(t_{1}-t_{2}\right)\right]^{1 / 4}\left[\xi+i\left(t_{1}-t_{3}\right)\right]^{1 / 4}\left[\xi+i\left(t_{2}-t_{4}\right)\right]^{1 / 4}\left[\xi+i\left(t_{3}-t_{4}\right)\right]^{1 / 4}} .
\end{aligned}
$$

Here $F_{12}, F_{13}$, and $F_{14}$ are the three characteristic functions appearing in the fourth-order correlation functions. For an Abelian state, they usually appear in quasi-symmetric combinations and exchanging two of the times alters various phase factors, which is a characteristic of fractional statistics. For the current non-Abelian case, however, exchanging two of the times not only alters phase factors but can also change the form of the correlation function from one of the characteristic functions to another. This is a special feature of non-Abelian statistics. $^{14}$

\section{APPENDIX D: SECOND-ORDER CORRECTION TO $\left\langle\sigma^{x}(t) \sigma^{x}(0)\right\rangle_{c}$}

Because our ultimate goal is to compute the qubit susceptibility, we are interested in the correlator with $t>0$ in the long-time limit $t \rightarrow \infty$. Let us first recall the perturbative part of the Hamiltonian Eq.(27) in the interaction picture:

$$
V\left(t, x_{0}\right)=-\frac{\delta}{2} \mu(t)\left[\tau^{+}(t)+\tau^{-}(t)\right] .
$$

Since the vortex tunneling in or out of the superconducting ring directly couples to the disorder field of the Ising model $\sigma^{x}(t)=\mu(t) \tau^{x}(t)$ in the transformed basis, the evaluation of the second-order correction for the correlator $\left\langle\sigma^{x}(t) \sigma^{x}(0)\right\rangle$ requires the knowledge of the four-point correlation function derived in Appendix C.

We expand the $S$ and $S^{\dagger}$ matrices in (31) to second order with insertions at times $t_{1}$ and $t_{2}$. Nonzero contributions to the correlator come from three regions: $\mathrm{A}, t>0>t_{1}>t_{2}$; $\mathrm{B}$, $t>t_{1}>0>t_{2}$; and $\mathrm{C}, t>t_{1}>t_{2}>0$. These three regions are shown in Fig. 3. In what follows, we will evaluate the second-order contributions from each region in the long-time limit.

\section{Region A: $t>0>t_{1}>t_{2}$}

The contribution from region $\mathrm{A}$ is given by

$$
\left\langle\sigma^{x}(t) \sigma^{x}(0)\right\rangle_{A}^{(2)}=(-i)^{2} \int_{-\infty}^{0} d t_{1} \int_{-\infty}^{t_{1}} d t_{2} I_{A},
$$

with the integrand

$$
\begin{aligned}
I_{A}= & +\left\langle\sigma^{x}(t) \sigma^{x}(0) V_{1} V_{2}\right\rangle_{0}+\left\langle V_{2} V_{1} \sigma^{x}(t) \sigma^{x}(0)\right\rangle_{0} \\
& -\left\langle V_{2} \sigma^{x}(t) \sigma^{x}(0) V_{1}\right\rangle_{0}-\left\langle V_{1} \sigma^{x}(t) \sigma^{x}(0) V_{2}\right\rangle_{0},
\end{aligned}
$$

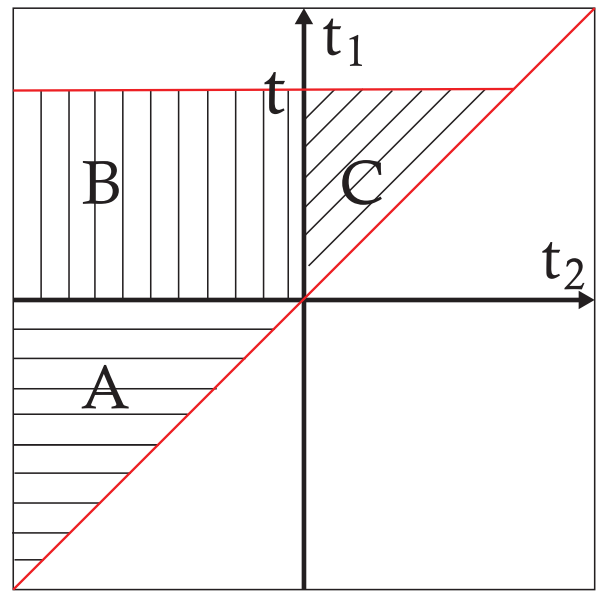

FIG. 3. (Color online) The integral domains for regions A, B, and $\mathrm{C}$ in the $t_{1}$ and $t_{2}$ coordinates used in Appendix D. 
where $V_{i} \equiv V\left(t_{i}\right)$ is a shorthand notation. The plus and minus signs come from the location of the insertions. The plus sign corresponds to having both insertions located on the same branch (either forward $S$ or backward $S^{\dagger}$ ) while the minus sign corresponds to the situation where the two insertions are located on different branches.

Because only certain orderings of insertions of raising and lowering operators $\tau^{+}$or $\tau^{-}$, coming both from the interaction term of Eq. (D1) and the $\tau^{x}$, give nonvanishing contributions, the integrand is given by

$$
\begin{aligned}
\left(\frac{2}{\delta}\right)^{2} I_{A}= & +e^{-i \varepsilon t} e^{i \varepsilon\left(t_{2}-t_{1}\right)}\left\langle\mu(t) \mu(0) \mu\left(t_{1}\right) \mu\left(t_{2}\right)\right\rangle \\
& +e^{-i \varepsilon t} e^{i \varepsilon\left(t_{1}-t_{2}\right)}\left\langle\mu\left(t_{2}\right) \mu\left(t_{1}\right) \mu(t) \mu(0)\right\rangle \\
& -e^{+i \varepsilon t} e^{i \varepsilon\left(t_{1}-t_{2}\right)}\left\langle\mu\left(t_{2}\right) \mu(t) \mu(0) \mu\left(t_{1}\right)\right\rangle \\
& -e^{+i \varepsilon t} e^{i \varepsilon\left(t_{2}-t_{1}\right)}\left\langle\mu\left(t_{1}\right) \mu(t) \mu(0) \mu\left(t_{2}\right)\right\rangle .
\end{aligned}
$$

Here, the four-point correlation function can be read off from Eq. (C6) and simplified using the identity of Eq.(C3). Remarkably, these correlators have the same time-dependence function and differ only by phase factors. This feature is characteristic also to regions B and C. After some algebra, the integrand simplifies to

$$
\begin{aligned}
I_{A}= & 2\left(\frac{\delta}{2}\right)^{2} e^{-i \pi / 8}\left(e^{-i \varepsilon t}-e^{+i \varepsilon t}\right) \\
& \times \operatorname{Re}\left\{e^{i \varepsilon\left(t_{2}-t_{1}\right)} \frac{\left(t-t_{1}\right)^{1 / 4}\left(-t_{2}\right)^{1 / 4} e^{-i \pi / 8}}{t^{1 / 4}\left(t-t_{2}\right)^{1 / 4}\left(-t_{1}\right)^{1 / 4}\left(t_{1}-t_{2}\right)^{1 / 4}}\right\} .
\end{aligned}
$$

To evaluate the integral of Eq. (D2), we first simplify it by introducing new variables such that $t_{1}=-t T$ and $t_{2}=-t(T+\tau)$ with the new integrating domain $0<\tau<\infty$ and $0<T<\infty$. The second-order correction from region $\mathrm{A}$ becomes

$$
\begin{aligned}
\left\langle\sigma^{x}(t) \sigma^{x}(0)\right\rangle_{A}^{(2)}= & i t^{3 / 2} \delta^{2} e^{-i \pi / 8} \sin (\varepsilon t) \\
& \times \operatorname{Re}\left\{e^{-i \pi / 8} \int_{0}^{\infty} d \tau \frac{e^{-\varepsilon t(\eta+i) \tau}}{\tau^{1 / 4}}\right. \\
& \left.\times \int_{0}^{\infty} d T \frac{e^{-2 \eta \varepsilon t T}(1+T)^{1 / 4}(T+\tau)^{1 / 4}}{(1+T+\tau)^{1 / 4} T^{1 / 4}}\right\},
\end{aligned}
$$

where we have introduced a regularization factor $\exp \left(\varepsilon \eta t_{i}\right)$, with $\eta \rightarrow 0^{+}$.

The integral in Eq. (D6) will not generate any oscillatory dependence but is divergent when both $T$ and $\tau$ are large. It is thus convenient to separate the algebraic part of the integrand into three parts:

$$
\begin{gathered}
I_{A_{1}}=\frac{(1+T)^{1 / 4}(T+\tau)^{1 / 4}}{(1+T+\tau)^{1 / 4}(T \tau)^{1 / 4}}-\frac{1}{\tau^{1 / 4}}-\frac{\tau^{3 / 4}}{4(T+\tau)(1+T)}, \\
I_{A_{2}}=\frac{1}{\tau^{1 / 4}}, \quad I_{A_{3}}=\frac{\tau^{3 / 4}}{4(T+\tau)(1+T)} .
\end{gathered}
$$

Combined with the exponential prefactor, the integration of $I_{A_{1}}$ is regular, the integral of $I_{A_{2}}$ diverges linearly, and that of $I_{A_{3}}$ diverges logarithmically.

Integrating $I_{A_{2}}$ with all the exponential prefactors gives

$$
\begin{aligned}
\int & \frac{e^{-i \pi / 8} e^{-\varepsilon t(\eta+i) \tau} e^{-2 \eta \varepsilon t T}}{\tau^{1 / 4}} d \tau d T \\
= & \frac{e^{-i \pi / 8} \Gamma\left(\frac{3}{4}\right)}{2 \eta(\varepsilon t)^{7 / 4}(i+\eta)^{3 / 4}} \propto \frac{1}{(\varepsilon t)^{7 / 4}}\left[-i \frac{\Gamma\left(\frac{3}{4}\right)}{2 \eta}\right. \\
& \left.+\frac{3 \Gamma\left(\frac{3}{4}\right)}{8}+\mathcal{O}(\eta)\right], \quad \eta \rightarrow 0^{+}
\end{aligned}
$$

Since the the linear long-time divergence is purely imaginary, it does not contribute to the correlation function.

In the long-time limit, the integrals of $I_{A_{1}}$ and $I_{A_{3}}$ with all the exponential prefactors can be carried out to the lowest order in $1 /(\varepsilon t)$ and are given by

$$
\begin{gathered}
\int e^{-i \pi / 8} e^{-\varepsilon t(\eta+i) \tau} e^{-2 \eta \varepsilon t T} I_{A_{1}} d \tau d T \\
\sim \frac{\Gamma\left(\frac{7}{4}\right)(\pi-2(1+\log (8)))}{8(\varepsilon t)^{7 / 4}}, \\
\int e^{-i \pi / 8} e^{-\varepsilon t(\eta+i) \tau} e^{-2 \eta \varepsilon t T} I_{A_{3}} d \tau d T \\
\sim-\frac{\Gamma\left(\frac{7}{4}\right)\left(3 \log (8 \varepsilon t)-(3 \pi / \sqrt{2}) e^{-\frac{i \pi}{4}}+3 \gamma-4\right)}{12(\varepsilon t)^{7 / 4}} .
\end{gathered}
$$

We now add the real parts of the three integrals of Eqs. (D8), (D9), and (D10) and then multiply them with the prefactors in Eq. (D6). The result is the leading long-time contribution from region $\mathrm{A}$ to the qubit spin correlator:

$$
\left\langle\sigma^{x}(t) \sigma^{x}(0)\right\rangle_{A}^{(2)} \sim \frac{\delta^{2} e^{-i \pi / 8}\left(e^{i \varepsilon t}-e^{-i \varepsilon t}\right)}{2 t^{1 / 4} \varepsilon^{7 / 4}}\left\{\frac{\Gamma\left(\frac{7}{4}\right)(7+3 \pi-3 \gamma-18 \log (2)-3 \log (\varepsilon t))}{12}\right\} .
$$

In the long-time limit, the leading contribution is given by the term $\propto t^{-1 / 4} \log (\varepsilon t)$.

\section{Region B: $t>t_{1}>0>t_{2}$}

The contribution from region $\mathrm{B}$ is given by

$$
\left\langle\sigma^{x}(t) \sigma^{x}(0)\right\rangle_{B}^{(2)}=(-i)^{2} \int_{0}^{t} d t_{1} \int_{-\infty}^{0} d t_{2} I_{B}
$$

with the integrand

$$
\begin{aligned}
I_{B}= & +\left\langle\sigma^{x}(t) V_{1} \sigma^{x}(0) V_{2}\right\rangle_{0}+\left\langle V_{2} V_{1} \sigma^{x}(t) \sigma^{x}(0)\right\rangle_{0} \\
& -\left\langle V_{2} \sigma^{x}(t) V_{1} \sigma^{x}(0)\right\rangle_{0}-\left\langle V_{1} \sigma^{x}(t) \sigma^{x}(0) V_{2}\right\rangle_{0} .
\end{aligned}
$$

After ordering the the raising and lowering operators $\tau^{+}$or $\tau^{-}$ and using Eq. (C6), the integrand reads

$$
I_{B}=e^{-i \pi / 4} I_{B_{1}}+e^{+i \pi / 4} I_{B_{2}}-I_{B_{1}}^{*}-I_{B_{2}}^{*},
$$


where the two integrand functions are given by

$$
\begin{gathered}
I_{B_{1}}=\frac{\delta^{2} e^{-i \varepsilon t} e^{i \varepsilon\left(t_{1}+t_{2}\right)} t^{1 / 4}\left(t_{1}-t_{2}\right)^{1 / 4}}{4\left(t-t_{1}\right)^{1 / 4}\left(t-t_{2}\right)^{1 / 4}\left(t_{1}\right)^{1 / 4}\left(-t_{2}\right)^{1 / 4}}, \\
I_{B_{2}}=\frac{\delta^{2} e^{-i \varepsilon t} e^{i \varepsilon\left(t_{1}-t_{2}\right)} t^{1 / 4}\left(t_{1}-t_{2}\right)^{1 / 4}}{4\left(t-t_{1}\right)^{1 / 4}\left(t-t_{2}\right)^{1 / 4}\left(t_{1}\right)^{1 / 4}\left(-t_{2}\right)^{1 / 4}},
\end{gathered}
$$

with $x^{*}$ denoting complex conjugate of $x$. Again, the four-point correlators of $\mu$ 's in region B have the same functional form up to phase factors.

To evaluate the integral of $I_{B_{1}}$, we introduce new variables $x_{1}$ and $x_{2}$ with $t_{1}=t\left(1-x_{1}\right)$ and $t_{2}=-t x_{2}$ such that

$$
B_{1}=\int I_{B_{1}} d t_{1} d t_{2}=\frac{\delta^{2} t^{3 / 2}}{4} \int_{0}^{1} d x_{1} \int_{0}^{\infty} d x_{2} e^{-i \varepsilon t\left(x_{1}+x_{2}\right)} \frac{\left(1-x_{1}+x_{2}\right)^{1 / 4}}{\left(1-x_{1}\right)^{1 / 4}\left(1+x_{2}\right)^{1 / 4}\left(x_{1}\right)^{1 / 4}\left(x_{2}\right)^{1 / 4}} .
$$

We can then split the integral $B_{1}$ into an oscillatory contribution $B_{1}^{\mathrm{O}}$ and a nonoscillatory one $B_{1}^{\mathrm{NO}}$. Since the non-oscillatory contribution from Eq. (D15) is dominated by $x_{1} \sim x_{2} \approx 0$, we can expand the integrand around this point to get the leading contribution. Because we are interested in the correlator in the long-time limit, we then deform the integration contour in the complex plane such that both $x_{1}$ and $x_{2}$ change from 0 to $-i \infty$. The leading nonoscillatory contribution is given by

$$
B_{1}^{\mathrm{NO}} \sim \frac{\delta^{2} t^{3 / 2}}{4} \int_{0}^{-i \infty} d x_{1} \int_{0}^{-i \infty} d x_{2} e^{-i \varepsilon t\left(x_{1}+x_{2}\right)}\left(\frac{1}{\left(x_{1} x_{2}\right)^{1 / 4}}+\frac{\left(x_{1} x_{2}\right)^{3 / 4}}{4}\right)=\frac{\delta^{2} \Gamma\left(\frac{3}{4}\right)^{2} e^{i \pi / 4}}{4 \varepsilon^{3 / 2}}\left(-1+\frac{9}{64 \varepsilon^{2} t^{2}}\right) .
$$

The oscillatory contribution $B_{1}^{\mathrm{O}}$ is dominated by $x_{1} \approx 1$ and $x_{2} \approx 0$; we can thus expand the integrand around this point to get the leading contribution. Again we are interested in the correlator in the long-time limit and thus deform the integration contour such that $x_{1}$ varies from $1-i \infty$ to 1 and $x_{2}$ varies from 0 to $-i \infty$. After these transformations $B_{1}^{\mathrm{O}}$ evaluates to

$$
B_{1}^{\mathrm{O}} \sim \frac{\delta^{2} t^{3 / 2} e^{-i \varepsilon t}}{4} \int_{-i \infty}^{0} d u_{1} \int_{0}^{-i \infty} d x_{2} e^{-i \varepsilon t\left(u_{1}+x_{2}\right)} \frac{\left(x_{2}-u_{1}\right)^{1 / 4}\left(-u_{1}\right)^{1 / 4}}{u_{1} x_{2}^{1 / 4}}=\frac{\delta^{2} e^{-i \varepsilon t}}{4 \varepsilon^{7 / 4}}\left(\frac{\cos \left(\frac{\pi}{8}\right) \Gamma\left(\frac{5}{8}\right) \Gamma\left(\frac{3}{4}\right) \Gamma\left(\frac{7}{4}\right)}{\sqrt{2} t^{1 / 4} \Gamma\left(\frac{11}{8}\right)}\right),
$$

where $u_{1}=x_{1}-1$. Summing up, the leading contributions to $B_{1}$ are

$$
B_{1}=\frac{\delta^{2}}{4 \varepsilon^{3 / 2}}\left\{\Gamma\left(\frac{3}{4}\right)^{2} e^{i \pi / 4}\left(-1+\frac{9}{64 \varepsilon^{2} t^{2}}\right)+e^{-i \varepsilon t} \frac{\cos \left(\frac{\pi}{8}\right) \Gamma\left(\frac{5}{8}\right) \Gamma\left(\frac{3}{4}\right) \Gamma\left(\frac{7}{4}\right)}{\sqrt{2} \Gamma\left(\frac{11}{8}\right)(\varepsilon t)^{1 / 4}}\right\} .
$$

The leading nonoscillatory contribution of $B_{1}$ is a constant while the leading oscillatory contribution has a power-law decay $\propto$ $t^{-1 / 4}$.

To integrate $I_{B_{2}}$, we again use the variables $t_{1}=t\left(1-x_{1}\right)$ and $t_{2}=-t x_{2}$ such that

$$
B_{2}=\int I_{B_{2}} d t_{1} d t_{2}=\frac{\delta^{2} t^{3 / 2}}{4} \int_{0}^{1} d x_{1} \int_{0}^{\infty} d x_{2} e^{-i \varepsilon t\left(x_{1}-x_{2}\right)} \frac{\left(1-x_{1}+x_{2}\right)^{1 / 4}}{\left(x_{1}\right)^{1 / 4}\left(1+x_{2}\right)^{1 / 4}\left(1-x_{1}\right)^{1 / 4}\left(x_{2}\right)^{1 / 4}} .
$$

Once again, the nonoscillatory contribution is dominated by $x_{1} \sim x_{2} \approx 0$. We expand the algebraic part of the integrand around $x_{1}=x_{2}=0$, deform the integration contour such that $x_{1}$ runs from 0 to $-i \infty$ and $x_{2}$ from 0 to $i \infty$, and get

$$
B_{2}^{\mathrm{NO}} \sim \frac{\delta^{2} t^{3 / 2}}{4} \int_{0}^{-i \infty} d x_{1} \int_{0}^{i \infty} d x_{2} e^{-i \varepsilon t\left(x_{1}-x_{2}\right)}\left\{\frac{1}{x_{1}^{1 / 4} x_{2}^{1 / 4}}+\frac{x_{1}^{3 / 4} x_{2}^{3 / 4}}{4}\right\}=\frac{\delta^{2} \Gamma\left(\frac{3}{4}\right)^{2}}{4 \varepsilon^{3 / 2}}\left(1+\frac{9}{64 \varepsilon^{2} t^{2}}\right) .
$$

To evaluate the oscillatory part $B_{2}^{\mathrm{O}}$ of $B_{2}$, we expand the integrand around $x_{1}=1$ and $x_{2}=0$ for the leading contribution. The necessary deformation of the integration contour is now given by $x_{1}$ changing from $1-i \infty$ to 1 and $x_{2}$ from 0 to $i \infty$. The leading oscillatory contribution from Eq. (D18) is now given by

$$
B_{2}^{\mathrm{O}} \sim \frac{\delta^{2} t^{3 / 2}}{4} e^{-i \varepsilon t} \int_{-i \infty}^{0} d u_{1} \int_{0}^{i \infty} d x_{2} e^{-i \varepsilon t\left(u_{1}-x_{2}\right)}\left\{-\frac{\left(x_{2}-u_{1}\right)^{1 / 4}\left(-u_{1}\right)^{1 / 4}}{u_{1} x_{2}^{1 / 4}}\right\}=\frac{\delta^{2} \Gamma\left(\frac{3}{4}\right)^{2}}{4 \varepsilon^{7 / 4}} \frac{2 \Gamma\left(\frac{7}{4}\right)}{\sqrt{\pi} t^{1 / 4}} e^{i \frac{7 \pi}{8}} e^{-i \varepsilon t},
$$

with $u_{1}=x_{1}-1$. The final expression for $B_{2}$ is

$$
B_{2}=\frac{\delta^{2} \Gamma\left(\frac{3}{4}\right)^{2}}{4 \varepsilon^{3 / 2}}\left(1+\frac{9}{64 \varepsilon^{2} t^{2}}+\frac{2 \Gamma\left(\frac{7}{4}\right) e^{i \frac{7 \pi}{8}}}{\sqrt{\pi}(\varepsilon t)^{1 / 4}} e^{-i \varepsilon t}\right) .
$$


Similar to $B_{1}$, the leading nonoscillatory contribution of $B_{2}$ is a constant, while the leading oscillatory contribution has a power-law decay $\sim t^{-1 / 4}$.

From Eqs. (D12) and (D14), the leading contributions to the qubit spin correlation function from region B is given by

$$
\begin{aligned}
& \left\langle\sigma^{x}(t) \sigma^{x}(0)\right\rangle_{B}^{(2)}=-\left(e^{-i \pi / 4} B_{1}+e^{i \pi / 4} B_{2}-B_{1}^{*}-B_{2}^{*}\right) \\
& =\frac{\delta^{2} \Gamma\left(\frac{3}{4}\right)^{2}}{2 \varepsilon^{3 / 2}}\left(1-\cos (\pi / 4)-\frac{9 i \sin (\pi / 4)}{64 \varepsilon^{2} t^{2}}+\frac{3 \cos \left(\frac{\pi}{8}\right) \Gamma\left(\frac{5}{8}\right)}{8 \sqrt{2} \Gamma\left(\frac{11}{8}\right)(\varepsilon t)^{1 / 4}}\left(e^{i \varepsilon t}-e^{-i(\varepsilon t+\pi / 4)}\right)+\frac{\Gamma\left(\frac{7}{4}\right) e^{i \pi / 8}}{\sqrt{\pi}(\varepsilon t)^{1 / 4}}\left(e^{-i \varepsilon t}-e^{i \varepsilon t}\right)\right) .
\end{aligned}
$$

\section{Region $\mathrm{C}: t>t_{1}>t_{2}>0$}

The integral in region $\mathrm{C}$ reads

$$
\left\langle\sigma^{x}(t) \sigma^{x}(0)\right\rangle_{C}^{(2)}=(-i)^{2} \int_{0}^{t} d t_{1} \int_{0}^{t_{1}} d t_{2} I_{C} .
$$

We calculate the integrand $I_{C}$ in a similar way to regions A and $\mathrm{B}$. We get

$$
I_{C}=\left(e^{-i \pi / 4}+1\right)\left(I_{C_{1}}-I_{C_{2}}\right),
$$

with the two integrand functions being

$$
\begin{aligned}
I_{C_{1}} & =\frac{\delta^{2} e^{-i \varepsilon t} e^{i \varepsilon\left(t_{1}-t_{2}\right)}\left(t-t_{2}\right)^{1 / 4}\left(t_{1}\right)^{1 / 4}}{4\left(t-t_{1}\right)^{1 / 4}(t)^{1 / 4}\left(t_{2}\right)^{1 / 4}\left(t_{1}-t_{2}\right)^{1 / 4}}, \\
I_{C_{2}} & =\frac{\delta^{2} e^{i \varepsilon t} e^{-i \varepsilon\left(t_{1}+t_{2}\right)}\left(t-t_{2}\right)^{1 / 4}\left(t_{1}\right)^{1 / 4}}{4\left(t-t_{1}\right)^{1 / 4}(t)^{1 / 4}\left(t_{2}\right)^{1 / 4}\left(t_{1}-t_{2}\right)^{1 / 4}} .
\end{aligned}
$$

To integrate $I_{C_{1}}$, we make the variable transformation: $t_{1}=$ $t(T+1 / 2+\tau / 2)$ and $t_{2}=t(T+1 / 2-\tau / 2)$. In terms of the new variables, the integral of $I_{C_{1}}$ reads

$$
C_{1}=\frac{\delta^{2} t^{3 / 2} e^{-i \varepsilon t}}{4} \int_{0}^{1} d \tau \frac{e^{i \varepsilon t \tau}}{\tau^{1 / 4}} \int_{-1 / 2+\tau / 2}^{1 / 2-\tau / 2} d T \frac{(1 / 2-T+\tau / 2)^{1 / 4}(1 / 2+T+\tau / 2)^{1 / 4}}{(1 / 2-T-\tau / 2)^{1 / 4}(1 / 2+T-\tau / 2)^{1 / 4}}
$$

The integration over $T$ can be carried out exactly with the result

$$
C_{1}=\frac{\delta^{2} t^{3 / 2} e^{-i \varepsilon t}}{4} \frac{2 \sqrt{\pi} \Gamma\left(\frac{3}{4}\right)}{\Gamma\left(\frac{1}{4}\right)} \int_{0}^{1} d \tau \frac{e^{i \varepsilon t \tau}}{\tau^{1 / 4}} \sqrt{1-\tau^{2}}{ }_{2} F_{1}\left(-\frac{1}{4}, \frac{1}{2} ; \frac{5}{4} ;\left(\frac{1-\tau}{1+\tau}\right)^{2}\right)
$$

where ${ }_{2} F_{1}(\alpha, \beta ; \gamma ; x)$ is the Gaussian hypergeometric function. ${ }^{50}$

We deform the integration contour in Eq. (D27) such that $\tau$ goes from 0 to $+i \infty$ and then back from $1+i \infty$ to 1 . The leading contribution in the long-time limit is dominated by the region near the real axis. The expansion around $x=0$ leads to an oscillatory contribution while the expansion around $x=1$ leads to a nonoscillatory contribution. To the lowest few orders, the asymptotic behavior in the long-time limit is given by

$$
C_{1} \sim+\frac{\delta^{2} \Gamma\left(\frac{3}{4}\right)^{2} e^{i \pi / 4}}{4 \varepsilon^{3 / 2}}\left(-1+\frac{9}{64 \varepsilon^{2} t^{2}}\right)+\frac{\delta^{2} e^{-i \varepsilon t}}{4}\left\{\frac{e^{3 i \pi / 8} \Gamma\left(\frac{3}{4}\right) t^{3 / 4}}{\varepsilon^{3 / 4}}+\frac{e^{7 i \pi / 8} \Gamma\left(\frac{7}{4}\right)(6 \log (\varepsilon t)-(6+3 i) \pi+6 \gamma-14+36 \log (2))}{12 \varepsilon^{7 / 4} t^{1 / 4}}\right\}
$$

The oscillatory contribution contains a power-law divergent $t^{3 / 4}$ term. As we discuss later, this term contributes to the shift of the resonant frequency and to the damping for the $\left\langle\sigma^{+}(t) \sigma^{-}(0)\right\rangle$ correlation function.

To integrate $I_{C_{2}}$, we first change the integration variables to $\tau$ and $T$ defined by $t_{1}=t(T+\tau / 2)$ and $t_{2}=t(T-\tau / 2)$ such that the integral separates into two parts:

$$
C_{2}=\frac{\delta^{2} t^{3 / 2} e^{i \varepsilon t}}{4}\left\{\int_{0}^{1 / 2} d T \int_{0}^{2 T} d \tau+\int_{1 / 2}^{1} d T \int_{0}^{2-2 T} d \tau\right\}\left(e^{-i 2 \varepsilon t T} \frac{(1-T+\tau / 2)^{1 / 4}(T+\tau / 2)^{1 / 4}}{(1-T-\tau / 2)^{1 / 4}(T-\tau / 2)^{1 / 4}(\tau)^{1 / 4}}\right) .
$$

After changing $T \rightarrow 1-T$ in the second integral and then introducing $X=2 T$, this equation simplifies to

$$
C_{2}=\frac{\delta^{2} t^{3 / 2}}{4} \operatorname{Re}\left\{e^{i \varepsilon t} \int_{0}^{1} d X e^{-i \varepsilon t X} \int_{0}^{X} d \tau \frac{(2-X+\tau)^{1 / 4}(X+\tau)^{1 / 4}}{(2-X-\tau)^{1 / 4}(X-\tau)^{1 / 4}(\tau)^{1 / 4}}\right\}
$$

Again, we deform the integration contour in the integral over $X$ with $X$ changing from 0 to $-i \infty$ and then from $1-i \infty$ to 1 . Now the oscillatory contribution comes from $X \sim 0$ while the nonoscillatory one comes from $X \sim 1$. 
By expanding the integrand around these two points, we get the leading contributions:

$$
C_{2}=\frac{\delta^{2} \Gamma\left(\frac{3}{4}\right)^{2}}{4 \varepsilon^{3 / 2}}\left(1+\frac{9}{64 \varepsilon^{2} t^{2}}-\frac{\left(e^{i \varepsilon t} e^{i \pi / 8}+e^{-i \varepsilon t} e^{-i \pi / 8}\right)}{\varepsilon^{1 / 4} t^{1 / 4}} \frac{{ }_{2} F_{1}\left(-\frac{1}{4}, \frac{3}{4} ; \frac{3}{2} ;-1\right) \Gamma\left(\frac{7}{4}\right)}{\sqrt{\pi}}\right) .
$$

The Gaussian hypergeometric function evaluates to ${ }_{2} F_{1}\left(-\frac{1}{4}, \frac{3}{4} ; \frac{3}{2} ;-1\right) \approx 1.102$.

From Eqs. (D24) and (D25), we obtain a contribution to the qubit correlation function from region C:

$$
\begin{aligned}
\left\langle\sigma^{x}(t) \sigma^{x}(0)\right\rangle_{C}^{(2)}= & -\left(e^{-i \pi / 4}+1\right)\left(C_{1}-C_{2}\right) \sim \frac{\delta^{2} \Gamma\left(\frac{3}{4}\right)^{2}}{2 \varepsilon^{3 / 2}}\left(1+\cos \left(\frac{\pi}{4}\right)-\frac{9 i \sin \left(\frac{\pi}{4}\right)}{64 \varepsilon^{2} t^{2}}\right)+\left(e^{-i \pi / 8} \frac{e^{-i \varepsilon t}}{t^{1 / 4}}\right) \frac{\delta^{2} \Gamma\left(\frac{3}{4}\right)}{4 \varepsilon^{7 / 4}}\left(1+e^{-i \pi / 4}\right)(-i \varepsilon t) \\
& +\frac{\delta^{2} e^{-i \varepsilon t} e^{-i \pi / 8}\left(e^{-i \pi / 4}+1\right)}{4 \varepsilon^{7 / 4} t^{1 / 4}}\left\{\frac{\Gamma\left(\frac{7}{4}\right)(6 \log (\varepsilon t)-(6+3 i) \pi+6 \gamma-14+36 \log (2))}{12}\right\} \\
& +\frac{\delta^{2} \Gamma\left(\frac{3}{4}\right)^{2}\left(e^{-i \pi / 4}+1\right)\left(e^{i \varepsilon t} e^{i \pi / 8}+e^{-i \varepsilon t} e^{-i \pi / 8}\right)}{4 \varepsilon^{7 / 4} t^{1 / 4}} \frac{{ }_{2} F_{1}\left(-\frac{1}{4}, \frac{3}{4} ; \frac{3}{2} ;-1\right) \Gamma\left(\frac{7}{4}\right)}{\sqrt{\pi}}
\end{aligned}
$$

\section{Final result for $\left\langle\sigma^{x}(t) \sigma^{x}(0)\right\rangle_{c}^{(2)}$}

The second-order correction to the correlation function $\left\langle\sigma^{x}(t) \sigma^{x}(0)\right\rangle_{c}^{(2)}$ can be obtained by adding up the contributions from all three regions, given by Eqs. (D11), (D23), and (D32) and then subtracting $\left\langle\sigma^{x}\right\rangle^{2}$ as calculated in Eq. (40). The full expression for the correlator in the long-time limit is

$$
\begin{aligned}
& \left\langle\sigma^{x}(t) \sigma^{x}(0)\right\rangle_{c}^{(2)}=-i \frac{9 \delta^{2} \Gamma\left(\frac{3}{4}\right)^{2}}{64 \varepsilon^{3 / 2}} \frac{\sin \left(\frac{\pi}{4}\right)}{\varepsilon^{2} t^{2}}+e^{-i \pi / 8} \frac{e^{-i \varepsilon t}}{t^{1 / 4}} \frac{\delta^{2} \Gamma\left(\frac{3}{4}\right)}{4 \varepsilon^{7 / 4}}\left(1+e^{-i \pi / 4}\right)(-i \varepsilon t)+\frac{\delta^{2} \Gamma\left(\frac{7}{4}\right) \log (\varepsilon t) e^{-i \pi / 8}}{8 \varepsilon^{7 / 4} t^{1 / 4}}\left\{\left(2+e^{-i \pi / 4}\right) e^{-i \varepsilon t}-e^{i \varepsilon t}\right\} \\
& -\frac{\delta^{2} \Gamma\left(\frac{7}{4}\right) e^{-i \pi / 8} e^{-i \varepsilon t}}{4 \varepsilon^{7 / 4} t^{1 / 4}}\left\{\left(2+e^{-i \pi / 4}\right) \frac{(7+3 \pi-3 \gamma-18 \log (2))}{6}+\left(e^{i \pi / 4}+e^{i \pi / 2}\right) \frac{\pi}{4}\right\} \\
& +\frac{\delta^{2} \Gamma\left(\frac{3}{4}\right)^{2} \Gamma\left(\frac{7}{4}\right) e^{-i \varepsilon t}}{4 \varepsilon^{7 / 4} t^{1 / 4}}\left(\frac{2 e^{i \pi / 8}}{\sqrt{\pi}}-\frac{3 \cos \left(\frac{\pi}{8}\right) \Gamma\left(\frac{5}{8}\right) e^{-i \pi / 4}}{4 \sqrt{2} \Gamma\left(\frac{11}{8}\right) \Gamma\left(\frac{7}{4}\right)}+\frac{{ }_{2} F_{1}\left(-\frac{1}{4}, \frac{3}{4} ; \frac{3}{2} ;-1\right)\left(e^{-i 3 \pi / 8}+e^{-i \pi / 8}\right)}{\sqrt{\pi}}\right) \\
& +\frac{\delta^{2} \Gamma\left(\frac{3}{4}\right)^{2} e^{i \varepsilon t}}{4 \varepsilon^{7 / 4} t^{1 / 4}}\left\{\frac{[7+3 \pi-3 \gamma-18 \log (2)] e^{-i \pi / 8}}{8 \Gamma\left(\frac{3}{4}\right)}+\frac{3 \cos \left(\frac{\pi}{8}\right) \Gamma\left(\frac{5}{8}\right)}{4 \sqrt{2} \Gamma\left(\frac{11}{8}\right)}\right. \\
& \left.-\frac{2 \Gamma\left(\frac{7}{4}\right) e^{i \pi / 8}}{\sqrt{\pi}}+\frac{2 \cos \left(\frac{\pi}{8}\right)_{2} F_{1}\left(-\frac{1}{4}, \frac{3}{4} ; \frac{3}{2} ;-1\right) \Gamma\left(\frac{7}{4}\right)}{\sqrt{\pi}}\right\} \text {. }
\end{aligned}
$$

This result agrees well with the numerical evaluation of the integral. A power-law divergence $\sim t^{3 / 4}$ and a logarithmic contribution $\sim \log (\varepsilon t) / t^{1 / 4}$ dominate the long-time behavior of the correlator. However, this logarithmic contribution will be cut off either by the induced damping or by a finite temperature.

A heuristic way to see that the term diverging as $t^{3 / 4}$ corresponds to the self-energy correction is to add it to the zeroth-order correlator of $\left\langle\sigma^{x}(t) \sigma^{x}(0)\right\rangle$ given by Eq.(41). The sum of these two terms equals to

$$
\begin{gathered}
e^{-i \pi / 8} \frac{e^{-i \varepsilon t}}{t^{1 / 4}}\left(1-i \frac{\delta^{2} \Gamma\left(\frac{3}{4}\right)}{4 \varepsilon^{3 / 4}}\left(2 \cos ^{2}\left(\frac{\pi}{8}\right)-i \frac{1}{\sqrt{2}}\right) t\right) \\
=e^{-i \pi / 8} \frac{e^{-i \varepsilon t}}{t^{1 / 4}}\left(1-i\left(v-i \frac{\gamma}{2}\right) t\right),
\end{gathered}
$$

with $v$ and $\gamma$ the same as in Eq. (51). It then becomes apparent that Eq. (D34) is exactly the expansion of the renormalized correlator of Eq. (55) to the second order in $\delta$ :

$$
\frac{e^{-i \pi / 8}}{t^{1 / 4}} e^{-i(\varepsilon+\nu) t-\gamma t / 2} \text {. }
$$

We thus conclude that the explicit evaluation of the higherorder correction gives a result consistent with the self-energy calculation.

\section{Comments on leading contributions of higher orders}

The leading contribution to the second order corrections comes from region $\mathrm{C}$ with the integration of $C_{1}$ when the integration variable $\tau$ is around $\tau=0$; cf. Eq. (D26). Since $\tau=\left(t_{1}-t_{2}\right) / t$, this expansion to the zeroth order is equivalent to making an operator product expansion of $\mu\left(t_{1}\right) \mu\left(t_{2}\right)$ for $t_{1} \approx t_{2}$ in the four-point correlation function of $\mu$ operators. ${ }^{41}$ In the $n$th order of perturbation theory with insertion times $t_{1}, \ldots, t_{n}$, we expect that the most divergent contribution arises when all the insertion times belong to the interval 
$[0, t]$. By ordering the times $t_{1}>t_{2}>\cdots>t_{n}$ and using the operator product expansion for the pairs $\mu_{t_{2 i-1}} \mu_{t_{2 i}}$ for $i=1, \ldots, n / 2$, we get a perturbative structure resembling
Wick's theorem. The resummation of these terms would give the contributions for the self-energy which we calculated in Sec. VI A.
${ }^{1}$ G. Moore and N. Read, Nucl. Phys. B 360, 362 (1991).

${ }^{2}$ P. Fendley, M. P. A. Fisher, and C. Nayak, Phys. Rev. B 75, 045317 (2007).

${ }^{3}$ B. Rosenow, B. I. Halperin, S. H. Simon, and A. Stern, Phys. Rev. Lett. 100, 226803 (2008).

${ }^{4}$ B. Rosenow, B. I. Halperin, S. H. Simon, and A. Stern, Phys. Rev. B 80, 155305 (2009).

${ }^{5}$ N. Read and D. Green, Phys. Rev. B 61, 10267 (2000).

${ }^{6}$ G. E. Volovik, JETP Lett. 70, 609 (1999).

${ }^{7}$ D. A. Ivanov, Phys. Rev. Lett. 86, 268 (2001).

${ }^{8}$ A. Y. Kitaev, Ann. Phys. 303, 2 (2003).

${ }^{9}$ C. Nayak, S. H. Simon, A. Stern, M. Freedman, and S. Das Sarma, Rev. Mod. Phys. 80, 1083 (2008).

${ }^{10}$ S. Das Sarma, M. Freedman, and C. Nayak, Phys. Rev. Lett. 94, 166802 (2005).

${ }^{11}$ A. Stern and B. I. Halperin, Phys. Rev. Lett. 96, 016802 (2006).

${ }^{12}$ P. Bonderson, A. Kitaev, and K. Shtengel, Phys. Rev. Lett. 96, 016803 (2006).

${ }^{13}$ E.-A. Kim, M. J. Lawler, S. Vishveshwara, and E. Fradkin, Phys. Rev. B 74, 155324 (2006).

${ }^{14}$ C. Bena and C. Nayak, Phys. Rev. B 73, 155335 (2006).

${ }^{15} \mathrm{G}$. E. Volovik, The Universe in a Helium Droplet (Clarendon, Oxford, 2003).

${ }^{16}$ A. Y. Kitaev, Phys. Usp. 44, 131 (2001).

${ }^{17}$ L. Fu and C. L. Kane, Phys. Rev. Lett. 100, 096407 (2008).

${ }^{18}$ L. Fu and C. L. Kane, Phys. Rev. Lett. 102, 216403 (2009).

${ }^{19}$ A. R. Akhmerov, J. Nilsson, and C. W. J. Beenakker, Phys. Rev. Lett. 102, 216404 (2009).

${ }^{20}$ J. D. Sau, R. M. Lutchyn, S. Tewari, and S. Das Sarma, Phys. Rev. Lett. 104, 040502 (2010).

${ }^{21}$ J. Alicea, Phys. Rev. B 81, 125318 (2010).

${ }^{22}$ A. C. Potter and P. A. Lee, Phys. Rev. Lett. 105, 227003 (2010).

${ }^{23}$ M. Duckheim and P. W. Brouwer, Phys. Rev. B 83, 054513 (2011).

${ }^{24}$ S. B. Chung, H.-J. Zhang, X.-L. Qi, and S.-C. Zhang, e-print arXiv:1011.6422.

${ }^{25}$ J. Nilsson and A. R. Akhmerov, Phys. Rev. B 81, 205110 (2010).

${ }^{26}$ J. D. Sau, S. Tewari, and S. Das Sarma, e-print arXiv:1004.4702.

${ }^{27}$ Yu. Makhlin, G. Schön, and A. Shnirman, Rev. Mod. Phys. 73, 357 (2001).

${ }^{28}$ F. Hassler, A. R. Akhmerov, C.-Y. Hou, and C. W. J. Beenakker, New J. Phys. 12, 125002 (2010).

${ }^{29}$ J. Alicea, Y. Oreg, G. Refael, F. von Oppen, and M. P. A. Fisher, Nat. Phys. 7, 412 (2011).

${ }^{30}$ J. D. Sau, S. Tewari, and S. Das Sarma, Phys. Rev. A 82, 052322 (2010).

${ }^{31}$ I. Chiorescu, Y. Nakamura, C. J. P. M. Harmans, and J. E. Mooij, Science 299, 1869 (2003).

${ }^{32} \mathrm{M}$. Tinkham, Introduction to Superconductivity, 2nd ed. (McGrawHill, New York, 1996).

${ }^{33}$ J. B. Zuber and C. Itzykson, Phys. Rev. D 15, 2875 (1977).
${ }^{34}$ S. Sachdev, Quantum Phase Transition, (Cambridge University Press, Cambridge, 1999).

${ }^{35}$ E. Fradkin and L. Susskind, Phys. Rev. D 17, 2637 (1978).

${ }^{36}$ J. B. Kogut, Rev. Mod. Phys. 51, 659 (1979).

${ }^{37}$ In the present paper, the Jordan-Wigner transformation of Eq.(11) is introduced for the Ising spin fields $s^{x}$. If the transformation is introduced for the disorder field, as in Refs. 41 and 42 , one should interchange the Ising spin field and the disorder field in our discussion.

${ }^{38}$ C. L. Kane and M. P. A. Fisher, Phys. Rev. B 46, 7268 (1992).

${ }^{39}$ C. L. Kane and M. P. A. Fisher, Phys. Rev. B 46, 15233 (1992).

${ }^{40} \mathrm{P}$. Ginsparg, in Fields, Strings, and Critical Phenomena, Les Houches Lectures, edited by E. Brézin and J. Zinn-Justin (NorthHolland, Amsterdam, 1989).

${ }^{41}$ P. Di Francesco, P. Mathieu, and D. Sénéchal, Conformal Field Theory (Springer-Verlag, New York, 1997).

${ }^{42}$ D. Allen and D. Sénéchal, Phys. Rev. B 61, 12134 (2000).

${ }^{43}$ G. D. Mahan, Many-Particle Physics, 3rd ed. (Plenum, New York, 2000).

${ }^{44}$ C. Cohen-Tannoudji, J. Dupont-Roc, and G. Grynberg, AtomPhoton Photon Interactions: Basic Processes and Applications, (Wiley-VCH Verlag GmbH \& Co. KGaA, Weinheim, 2004).

${ }^{45}$ R. Shankar, Int. J. Mod. Phys. B 4, 2371 (1990).

${ }^{46}$ For a Lorentzian response, the susceptibility is given by $\chi(\omega) \propto$ $1 /[(\varepsilon+v-\omega)-i \gamma / 2]$ that leads to a phase change of $\pi$, compared with $3 \pi / 4$ in Eq. (58), across the resonance. Here, the decay rate can generally be expressed as $\gamma=\gamma_{0}+\gamma^{\prime}(T)$ with $\gamma^{\prime}(T) \propto T$ for high-temperature $T>\varepsilon^{44}$. In contrast to Eqs. (59) and (61), the resonance peak for a Lorentzian response thus scales as $1 / \gamma_{0}$ at zero temperature while it scales as $1 / T$ at finite temperature.

${ }^{47}$ For a topological insulator-based proposal with time-reversal symmetry in the bulk, cf. Ref. 18, the velocity of Majorana edge modes is further suppressed and is given by $v_{M} \sim v_{F}\left(\Delta / E_{F}\right)^{2}$ when $E_{F} \gg \Delta$. The cutoff energy for the linear dispersion is constrained to the region $\Lambda \sim \Delta^{2} / E_{F}$. In this case, we still get $a=\lambda_{F}$, the Fermi wavelength.

${ }^{48}$ V. E. Manucharyan, J. Koch, L. I. Glazman, and M. H. Devoret, Science 326, 113 (2009).

${ }^{49}$ V. E. Manucharyan, N. A. Masluk, A. Kamal, J. Koch, L. I. Glazman, and M. H. Devoret, e-print arXiv:1012.1928.

${ }^{50}$ M. Abramowitz and I. A. Stegun (eds.), Handbook of Mathematical Functions (Dover, New York, 1972).

${ }^{51}$ A. J. Leggett, S. Chakravarty, A. T. Dorsey, M. P. A. Fisher, A. Garg, and Zwerger, Rev. Mod. Phys. 59, 1 (1987).

${ }^{52}$ S. Coleman, Aspects of Symmetry (Cambridge University Press, Cambridge, 1985).

${ }^{53}$ The basis for the two-level system, $|\phi=0\rangle$ and $|\phi=2 \pi\rangle$, used in Appendix B directly denotes the superconductor phase difference across the junction. This basis is in turn different from the basis 10$\rangle$ and $|2 \pi\rangle$ defined in the main text, right above Eq. (9). They are, however, related by $|0\rangle=|\phi=0\rangle$ and $|2 \pi\rangle=P|\phi=2 \pi\rangle$. 